\title{
Moments of nucleon generalized parton distributions from lattice QCD
}

\author{
C. Alexandrou, ${ }^{1,2}$ J. Carbonell, ${ }^{3}$ M. Constantinou, ${ }^{1}$ P. A. Harraud, ${ }^{3}$ P. Guichon, ${ }^{4}$ K. Jansen, ${ }^{5}$ C. Kallidonis, ${ }^{1}$ \\ T. Korzec, ${ }^{1,6}$ and M. Papinutto ${ }^{3}$ \\ ${ }^{1}$ Department of Physics, University of Cyprus, Post Office Box 20537, 1678 Nicosia, Cyprus \\ ${ }^{2}$ Computation-based Science and Technology Research Center, Cyprus Institute, 20 Kavafi Street, 2121 Nicosia, Cyprus \\ ${ }^{3}$ Laboratoire de Physique Subatomique et Cosmologie, UJF/CNRS/IN2P3, 53 avenue des Martyrs, 38026 Grenoble, France \\ ${ }^{4}$ CEA-Saclay, IRFU/Service de Physique Nucléaire, 91191 Gif-sur-Yvette, France \\ ${ }^{5}$ NIC, DESY, Platanenallee 6, D-15738 Zeuthen, Germany \\ ${ }^{6}$ Institut für Physik, Humboldt Universität zu Berlin, Newtonstrasse 15, 12489 Berlin, Germany
}

(Received 9 April 2011; published 21 June 2011)

\begin{abstract}
We present results on the lower moments of the nucleon generalized parton distributions within lattice QCD using two dynamical flavors of degenerate twisted mass fermions. Our simulations are performed on lattices with three different values of the lattice spacings, namely, $a=0.089 \mathrm{fm}, a=0.070 \mathrm{fm}$, and $a=0.056 \mathrm{fm}$, allowing the investigation of cutoff effects. The volume dependence is examined using simulations on two lattices of spatial length $L=2.1 \mathrm{fm}$ and $L=2.8 \mathrm{fm}$. The simulations span pion masses in the range of 260 to $470 \mathrm{MeV}$. Our results are renormalized nonperturbatively, and the values are given in the $\overline{\mathrm{MS}}$ scheme at a scale $\mu=2 \mathrm{GeV}$. They are chirally extrapolated to the physical point in order to compare with the experiment. The consequences of these results on the spin carried by the quarks in the nucleon are investigated.
\end{abstract}

DOI: 10.1103/PhysRevD.83.114513

\section{INTRODUCTION}

Lattice QCD calculations of observables, related to the structure of baryons, are now carried out using simulations of the theory with parameters that are close enough to their physical values that a connection of lattice results to the experiment is facilitated. This is due to the fact that systematic uncertainties caused by a finite volume, a finite lattice cutoff, and unphysically high pion masses are becoming better controlled. Nowadays, a number of major collaborations are producing results on nucleon form factors and the first moments of structure functions close to the physical regime, both in terms of pion mass and with respect to the continuum limit [1-6].

The generalized parton distributions (GPDs) encode important information related to baryon structure [7-9]. They occur in several physical processes, such as deeply virtual Compton scattering and deeply virtual meson production. Their forward limit coincides with the usual parton distributions, and their first moments are related to the nucleon elastic form factors. Moreover, a combination of their second moments, known as Ji's sum rule [10], allows to determine the contribution of a specific parton to the nucleon angular momentum. In the context of the "proton spin puzzle," this has triggered an intense experimental activity [11-15].

The GPDs can be accessed in high-energy processes where QCD factorization applies, in which case the amplitude is the convolution of a hard perturbative kernel with the GPDs, as illustrated in Fig. 1. Generically, the GPDs are defined as matrix elements of bi-local operators separated by a lightlike interval. Because of the Wick rotation, such matrix elements cannot be computed directly on the
PACS numbers: 11.15.Ha, 12.38.-t, 12.38.Aw, 12.38.Gc

Euclidean lattice. Instead, one considers their Mellin moments, which, in principle, carry the same information.

If $\left|p^{\prime}\right\rangle$ and $|p\rangle$ are one-particle states, the twist-two GPDs, which are studied in this paper, are defined by the matrix element [16]

$$
\begin{aligned}
F_{\Gamma}\left(x, \xi, q^{2}\right)= & \frac{1}{2} \int \frac{d \lambda}{2 \pi} e^{i x \lambda}\left\langle p^{\prime}\right| \bar{\psi}(-\lambda n / 2) \\
& \times \Gamma \mathcal{P} e^{i g \int_{-\lambda / 2}^{\lambda / 2} d \alpha n \cdot A(n \alpha)} \psi(\lambda n / 2)|p\rangle,
\end{aligned}
$$

where $q=p^{\prime}-p, \xi=-n \cdot q / 2, x$ is the momentum fraction, and $n$ is a lightlike vector collinear to $P=$ $\left(p+p^{\prime}\right) / 2$ and such that $P \cdot n=1$. The gauge link $\mathcal{P} \exp (\ldots)$ is necessary for gauge invariance. In model calculations, it is often set to one, which amounts to working with QCD in the lightlike gauge $A \cdot n=0$; however, on the lattice, such a gauge fixing is not necessary.

In this work, we shall consider only the GPDs corresponding to a Dirac structure $\Gamma$ which conserves the quark chirality; that is, $\Gamma=\hbar$ and $\Gamma=h \gamma_{5}$. The associated matrix elements may be parametrized in the following way:

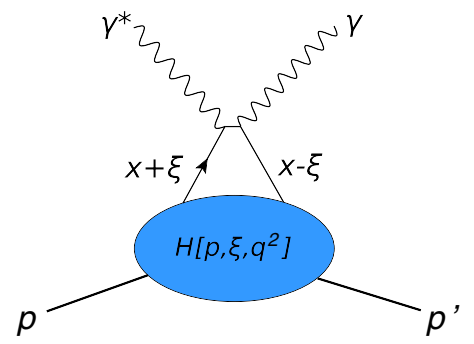

FIG. 1 (color online). "Handbag” diagram. 


$$
\begin{aligned}
F_{\varkappa}\left(x, \xi, q^{2}\right)= & \frac{1}{2} \bar{u}_{N}\left(p^{\prime}\right)\left[\not H\left(x, \xi, q^{2}\right)\right. \\
+ & \left.i \frac{n_{\mu} q_{\nu} \sigma^{\mu \nu}}{2 m_{N}} E\left(x, \xi, q^{2}\right)\right] u_{N}(p), \\
F_{\varkappa \gamma_{5}}\left(x, \xi, q^{2}\right)= & \frac{1}{2} \bar{u}_{N}\left(p^{\prime}\right)\left[\not \gamma_{5} \tilde{H}\left(x, \xi, q^{2}\right)\right. \\
& \left.+\frac{n \cdot q \gamma_{5}}{2 m_{N}} \tilde{E}\left(x, \xi, q^{2}\right)\right] u_{N}(p),
\end{aligned}
$$

where $u_{N}$ is a nucleon spinor and $H, E, \tilde{H}$, and $\tilde{E}$ are the twist-two chirality-even GPDs. In the forward limit, for which $\xi=0$ and $q^{2}=0$, they reduce to the ordinary parton distributions; namely, the longitudinal momentum $q(x)$ and the helicity $\Delta q(x)$ distributions are given by

$$
q(x)=H(x, 0,0) \text { and } \Delta q(x)=\tilde{H}(x, 0,0)
$$

The first few Mellin moments of these parton distributions are of particular interest:

$$
\begin{aligned}
\left\langle x^{n-1}\right\rangle_{q} & =\int_{-1}^{1} x^{n-1} q(x) d x, \\
\left\langle x^{n-1}\right\rangle_{\Delta q} & =\int_{-1}^{1} x^{n-1} \Delta q(x) d x .
\end{aligned}
$$

Since, as already mentioned, matrix elements of the lightcone operator, as defined in Eq. (1), cannot be extracted from correlators in Euclidean lattice QCD, the usual method is to proceed with an operator product expansion of this operator that leads to a tower of local operators given by

$$
\begin{gathered}
\mathcal{O}_{V}^{\mu_{1} \ldots \mu_{n}}=\bar{\psi} \gamma^{\left\{\mu_{1}\right.} i \stackrel{D}{D}^{\mu_{2}} \ldots i \stackrel{\leftrightarrow}{D}^{\left.\mu_{n}\right\}} \psi, \\
\mathcal{O}_{A}^{\mu_{1} \ldots \mu_{n}}=\bar{\psi} \gamma^{\left\{\mu_{1}\right.} i \stackrel{D^{\mu_{2}}}{\mu} \ldots i \stackrel{\leftrightarrow}{D}^{\left.\mu_{n}\right\}} \gamma_{5} \psi .
\end{gathered}
$$

The curly brackets represent a symmetrization over indices and the subtraction of traces. The computation of the matrix elements of these operators on the Euclidean lattice can be done with standard techniques. The case $n=1$ amounts to calculating the elastic form factors of the vector and axial-vector currents, and the results are reported in Refs. [4,17]. In this work, we concentrate on the $n=2$ moments, i.e., the matrix elements of operators with a single derivative. The matrix elements of these operators are parametrized in terms of the generalized form factors (GFFs) $A_{20}\left(q^{2}\right), B_{20}\left(q^{2}\right), C_{20}\left(q^{2}\right)$ and $\tilde{A}_{20}\left(q^{2}\right), \tilde{B}_{20}\left(q^{2}\right)$, according to

$$
\begin{aligned}
\left\langle N\left(p^{\prime}, s^{\prime}\right)\left|\mathcal{O}_{h}^{\mu \nu}\right| N(p, s)\right\rangle= & \bar{u}_{N}\left(p^{\prime}, s^{\prime}\right)\left[A_{20}\left(q^{2}\right) \gamma^{\{\mu} P^{\nu\}}\right. \\
& +B_{20}\left(q^{2}\right) \frac{i \sigma^{\{\mu \alpha} q_{\alpha} P^{\nu\}}}{2 m} \\
& \left.+C_{20}\left(q^{2}\right) \frac{1}{m} q^{\{\mu} q^{\nu\}}\right] u_{N}(p, s), \\
\left\langle N\left(p^{\prime}, s^{\prime}\right)\left|\mathcal{O}_{\not \gamma /}^{\mu \nu}\right| N(p, s)\right\rangle= & \bar{u}_{N}\left(p^{\prime}, s^{\prime}\right)\left[\tilde{A}_{20}\left(q^{2}\right) \gamma^{\{\mu} P^{\nu\}} \gamma^{5}\right. \\
& \left.+\tilde{B}_{20}\left(q^{2}\right) \frac{q^{\{\mu} P^{\nu\}}}{2 m} \gamma^{5}\right] u_{N}(p, s) .
\end{aligned}
$$

Note that the GFFs depend only on the squared momentum transfer $q^{2}=\left(p^{\prime}-p\right)^{2}$, which implies that the moments of the GPDs are polynomial in $\xi$. In the forward limit, we have $A_{20}(0)=\langle x\rangle_{q}$ and $\tilde{A}_{20}(0)=\langle x\rangle_{\Delta q}$, which are, respectively, the first moments of the unpolarized and polarized quark distributions. Knowing the GFFs, one can evaluate the quark contribution to the nucleon spin using Ji's sum rule: $J^{q}=\frac{1}{2}\left[A_{20}^{q}(0)+B_{20}^{q}(0)\right]$. Moreover, using the measured or calculated value of the quark helicity $\Delta \Sigma^{q}=g_{A}^{q}$, the decomposition $J^{q}=$ $\frac{1}{2} \Delta \Sigma^{q}+L^{q}$ allows to study the role of the quark orbital angular momentum $L^{q}$.

\section{LATTICE EVALUATION}

Twisted mass fermions [18] provide an attractive formulation of lattice QCD that allows for automatic $\mathcal{O}(a)$ improvement, infrared regularization of small eigenvalues, and fast dynamical simulations [19]. For the calculation of the moments of GPDs, which is the main focus of this work, the automatic $\mathcal{O}(a)$ improvement is particularly relevant, since it is achieved by tuning only one parameter in the action, requiring no further improvements on the operator level.

The action for two degenerate flavors of quarks in twisted mass QCD is given by

$$
S=S_{g}+\sum_{x} \bar{\chi}(x)\left[D_{W}+m_{\mathrm{crit}}+i \gamma_{5} \tau^{3} \mu\right] \chi(x),
$$

where $D_{W}$ is the Wilson Dirac operator, and we use the tree-level Symanzik improved gauge action $S_{g}$ [20]. The quark fields $\chi$ are in the so-called "twisted basis" obtained from the "physical basis" at maximal twist by a simple transformation:

$$
\psi=\frac{1}{\sqrt{2}}\left[\mathbb{1}+i \tau^{3} \gamma_{5}\right] \chi \quad \text { and } \quad \bar{\psi}=\bar{\chi} \frac{1}{\sqrt{2}}\left[\mathbb{1}+i \tau^{3} \gamma_{5}\right]
$$

We note that, in the continuum, this action is equivalent to the standard QCD action. As we pointed out, a crucial advantage is the fact that, by tuning a single parameter, namely, the bare untwisted quark mass to its critical value 
$m_{\text {crit }}$, a wide class of physical observables are automatically $\mathcal{O}(a)$ improved $[18,19,21]$. A disadvantage is the explicit flavor symmetry breaking. In a recent paper, we have checked that this breaking is small for the baryon observables under consideration in this work and for the lattice spacings that we use [22-26]. To extract the GFFs without needing to evaluate the disconnected contributions, we evaluate the nucleon matrix elements corresponding to the operators defined by

$$
\begin{aligned}
& \mathcal{O}_{V^{a}}^{\mu_{1} \ldots \mu_{n}}=\bar{\psi} \gamma^{\left\{\mu_{1}\right.} i D^{\mu_{2}} \ldots i D^{\left.\mu_{n}\right\}} \frac{\tau^{a}}{2} \psi, \\
& \mathcal{O}_{A^{a}}^{\mu_{1} \ldots \mu_{n}}=\bar{\psi} \gamma^{5} \gamma^{\left\{\mu_{1}\right.} i D^{\mu_{2}} \ldots i D^{\left.\mu_{n}\right\}} \frac{\tau^{a}}{2} \psi,
\end{aligned}
$$

where, from now on, we use the notations $\mathcal{O}_{V^{a}}^{\mu \ldots \mu_{n}}$ and $\mathcal{O}_{A^{a}}^{\mu \ldots \mu_{n}}$ to denote the vector and axial-vector operators with flavor index $a$. These matrix elements receive contributions only from the connected diagram for $a=1,2$ and up to $\mathcal{O}\left(a^{2}\right)$ for $a=3$ [27]. In particular, we consider the isovector combination with $a=3$, for which the form of the operators remains the same in the physical and twisted basis. In order to find the spin carried by each quark in the nucleon, we also analyze the isoscalar one-derivative vector and axial-vector operators. The latter receive contributions from disconnected fermion loops, which we neglect in this analysis. Simulations including a dynamical strange quark are also available within the twisted mass formulation. Comparison of the nucleon mass obtained with two dynamical flavors and the nucleon mass including a dynamical strange quark has shown negligible dependence on the dynamical strange quark [28]. We, therefore, expect the results on the nucleon moments to show little sensitivity on a dynamical strange quark. This is also confirmed by comparing our results to those where a dynamical strange quark is included.

In this work, we consider simulations at three values of the coupling constant with lattice spacings $0.056,0.07$, and $0.089 \mathrm{fm}$ determined from the nucleon mass. This enables us to obtain results in the continuum limit. We also examine finite size effects by comparing results on two lattices of spatial length $L=2.1 \mathrm{fm}$ and $L=2.8 \mathrm{fm}$ [29-31].

\section{A. Correlation functions}

The GFFs are extracted from dimensionless ratios of correlation functions. The two-point and three-point functions are defined by

$$
\begin{aligned}
& G\left(\vec{q}, t_{f}-t_{i}\right)=\sum_{\vec{x}_{f}} e^{-i\left(\vec{x}_{f}-\vec{x}_{i}\right) \cdot \vec{q}} \Gamma_{\beta \alpha}^{0}\left\langle J_{\alpha}\left(t_{f}, \vec{x}_{f}\right) \bar{J}_{\beta}\left(t_{i}, \vec{x}_{i}\right)\right\rangle, \\
& G^{\mu_{1} \cdots \mu_{n}}\left(\Gamma^{\nu}, \vec{q}, t-t_{i}\right)= \sum_{\vec{x}, \vec{x}_{f}} e^{i\left(\vec{x}-\vec{x}_{i}\right) \cdot \vec{q}} \Gamma_{\beta \alpha}^{\nu}\left\langle J_{\alpha}\left(t_{f}, \vec{x}_{f}\right)\right. \\
&\left.\times \mathcal{O}^{\mu_{1} \cdots \mu_{n}}(t, \vec{x}) \bar{J}_{\beta}\left(t_{i}, \vec{x}_{i}\right)\right\rangle,
\end{aligned}
$$

where we consider kinematics for which the final momentum $p_{f}=0$. We drop $t_{f}-t_{i}$ from the argument of the three-point function, since it will be kept fixed in our approach. The projection matrices $\Gamma^{0}$ and $\Gamma^{k}$ are given by

$$
\Gamma^{0}=\frac{1}{4}\left(\mathbb{1}+\gamma_{0}\right), \quad \Gamma^{k}=i \Gamma^{0} \gamma_{5} \gamma_{k}, \quad k=1,2,3 .
$$

The proton-interpolating field, written in the twisted basis at maximal twist, is given by

$$
\tilde{J}(x)=\frac{1}{\sqrt{2}}\left[\mathbb{1}+i \gamma_{5}\right] \epsilon^{a b c}\left[\tilde{u}^{a \top}(x) \mathcal{C} \gamma_{5} \tilde{d}^{b}(x)\right] \tilde{u}^{c}(x),
$$

where $\mathcal{C}$ is the charge conjugation matrix. We use Gaussian smeared quark fields [32,33] to increase the overlap with the proton state and decrease overlap with excited states. The smeared quark fields are given by

$$
\begin{aligned}
q_{\text {smear }}^{a}(t, \vec{x}) & =\sum_{\vec{y}} F^{a b}(\vec{x}, \vec{y} ; U(t)) q^{b}(t, \vec{y}), \\
F & =(\mathbb{1}+\alpha H)^{n}, \\
H(\vec{x}, \vec{y} ; U(t)) & =\sum_{i=1}^{3}\left[U_{i}(x) \delta_{x, y-\hat{\imath}}+U_{i}^{\dagger}(x-\hat{\imath}) \delta_{x, y+\hat{\imath}}\right] .
\end{aligned}
$$

We also apply APE smearing to the gauge fields $U_{\mu}$ entering the hopping matrix $H$. The parameters for the Gaussian smearing $\alpha$ and $n$ are optimized using the nucleon mass, as described in Ref. [6].

For correlators containing the isovector operators, the disconnected diagrams are zero up to lattice artifacts and can be safely neglected as we approach the continuum limit. The detailed investigation of volume and cutoff effects will be performed on isovector quantities, for which no contributions are neglected. They can be calculated by evaluating the connected diagram, shown schematically in Fig. 2. A standard approach to calculate the connected three-point function is using sequential inversions through the sink $[34,35]$. The creation operator is taken at a fixed position $\vec{x}_{i}=\overrightarrow{0}$ (source). The annihilation operator, at a later time $t_{f}$ (sink), carries momentum $\vec{p}^{\prime}=\overrightarrow{0}$. The current couples to a quark at an intermediate time $t$ and carries momentum $\vec{q}$. Translation invariance enforces $\vec{q}=-\vec{p}$ for our kinematics. At a fixed source-sink time separation, we obtain results for all possible momentum transfers and insertion times, as well as for any operator $\mathcal{O}^{\left\{\mu_{1} \cdots \mu_{n}\right\}}$,

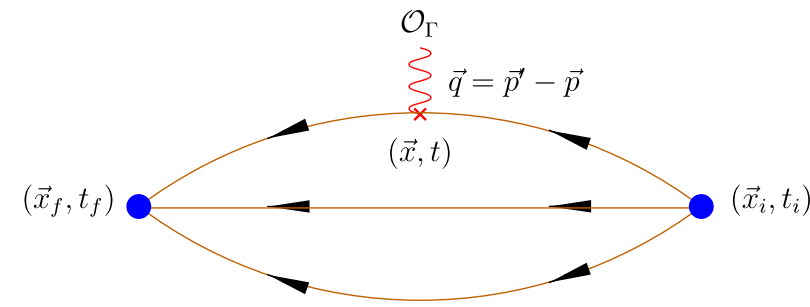

FIG. 2 (color online). Connected nucleon three-point function. 

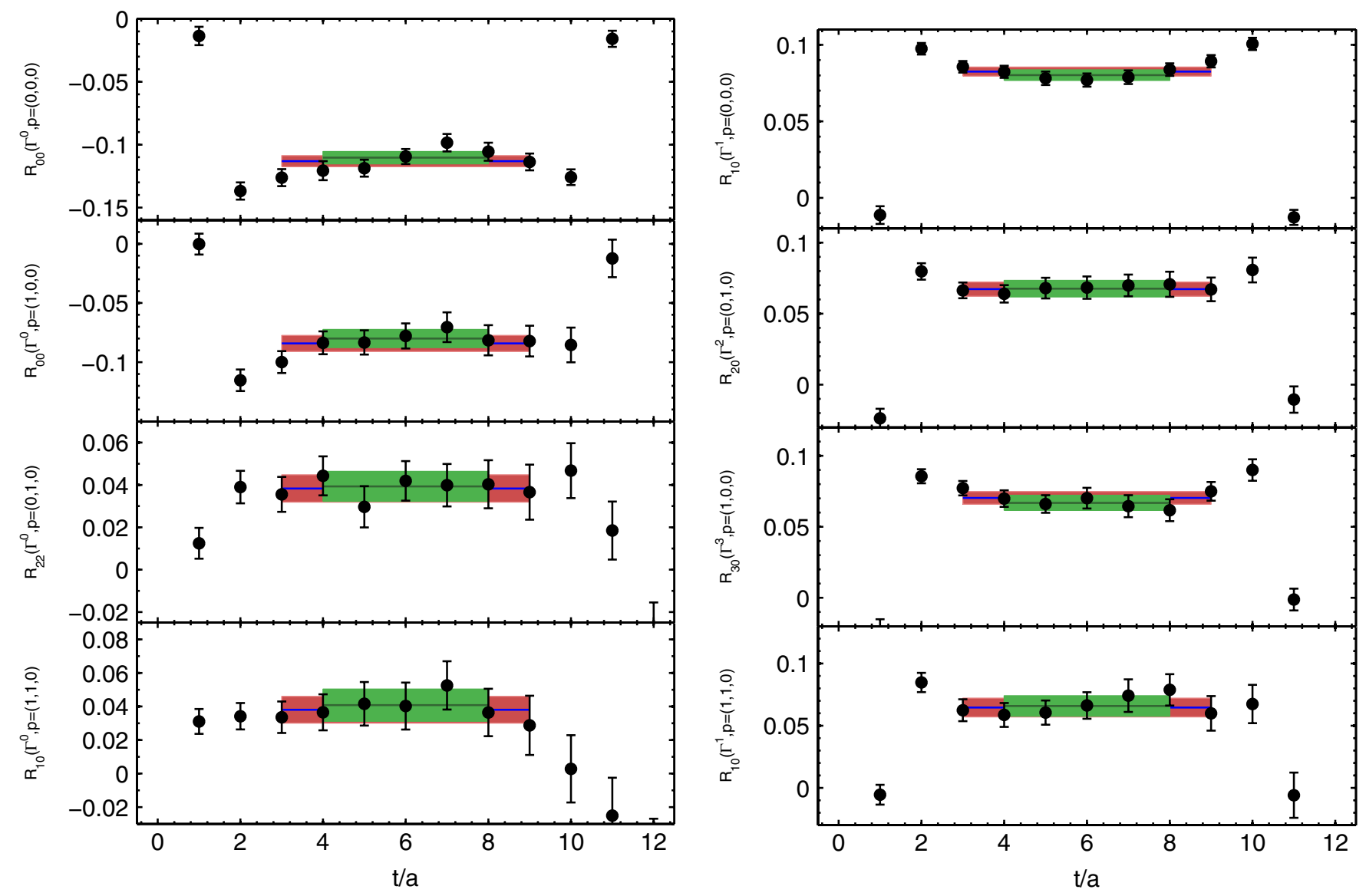

FIG. 3 (color online). Ratios for the one-derivative vector (left) and axial-vector (right) operator for a few exemplary choices of the momentum. The solid lines with the bands indicate the fitted plateau values with their jackknife errors. The extent of the bands shows the range of the plateaus.

with one set of sequential inversions per choice of the sink. We perform separate inversions for each one of the four projection matrices $\Gamma^{\mu}$ given in Eq. (15).

Using the two- and three-point functions of Eqs. (13)-(15) and considering only one-derivative operators, we form the ratio

$$
R^{\mu \nu}\left(\Gamma^{\lambda}, \vec{q}, t\right)=\frac{G^{\mu \nu}\left(\Gamma^{\lambda} \vec{q}, t\right)}{G\left(\overrightarrow{0}, t_{f}\right)} \sqrt{\frac{G\left(\vec{p}, t_{f}-t\right) G(\overrightarrow{0}, t) G\left(\overrightarrow{0}, t_{f}\right)}{G\left(\overrightarrow{0}, t_{f}-t\right) G(\vec{p}, t) G\left(\vec{p}, t_{f}\right)}},
$$

which is optimized because it does not contain potentially noisy two-point functions at large separations and because correlations between its different factors reduce the statistical noise. For sufficiently large separations $t_{f}-t$ and $t-t_{i}$, this ratio becomes time-independent (plateau region):

$$
\lim _{t_{f}-t \rightarrow \infty} \lim _{t-t_{i} \rightarrow \infty} R^{\mu \nu}\left(\Gamma^{\lambda}, \vec{q}, t\right)=\Pi^{\mu \nu}\left(\Gamma^{\lambda}, \vec{q}\right) .
$$

From the plateau values of the renormalized asymptotic ratio $\Pi\left(\Gamma^{\lambda}, \vec{q}\right)_{R}=Z \Pi\left(\Gamma^{\lambda}, \vec{q}\right)$, the generalized form factors can be extracted. The equations relating $\Pi\left(\Gamma^{\lambda}, \vec{q}\right)$ to the GFFs are given in Appendix A. All values of $\vec{q}$ resulting in the same $q^{2}$, the four choices of $\Gamma$, and the ten orientations $\mu$ and $\nu$ of the operator lead to an over-constrained system of equations which is solved in the least-squares sense via a singular value decomposition of the coefficient matrix. All quantities will be given in Euclidean space, with $Q^{2}=-q^{2}$ the Euclidean momentum transfer squared. The coefficients follow from the matrix-element decomposition given in Eq. (9) and may depend on the energy and mass of the nucleon, as well as on the initial spatial momentum $\vec{p}=-\vec{q}$ (see Appendix A). It turns out that both the operators with $\mu=\nu$ and $\mu \neq \nu$ are necessary to obtain all three one-derivative vector form factors. Since those two classes of operators on a lattice renormalize differently from each other [36], renormalization has to be carried out already on the level of the ratios. Although the one-derivative axial form factors can be extracted using only correlators with $\mu \neq \nu$, we use all combinations of $\mu$ and $\nu$ in order to decrease the statistical error.

Since we use sequential inversions through the sink, we need to fix the sink-source separation. The statistical errors on the three-point function are kept as small as possible by 
using the smallest value for the sink-source time separation that still ensures that the excited state contributions are sufficiently suppressed. We have tested different values of the sink-source time separation in the case of the form factors. We found that, taking $t_{f}-t_{i}=12 a$ and $t_{f}-t_{i}=14 a$, the results are consistent [6]. Therefore, for the nucleon matrix elements of the generalized form factors, we used throughout $t_{f}-t_{i}=12 a \sim 1 \mathrm{fm}$. For the lattices considered, we use the following values, which correspond to $t_{f}-t_{i} \sim 1 \mathrm{fm}$ :

$$
\begin{aligned}
& \beta=3.9:\left(t_{f}-t_{i}\right) / a=12, \\
& \beta=4.05:\left(t_{f}-t_{i}\right) / a=16, \\
& \beta=4.20:\left(t_{f}-t_{i}\right) / a=18 .
\end{aligned}
$$

In Fig. 3, we show representative plateaus for different momenta and indices $\mu$ and $\nu$. Fits using two different plateau ranges are shown, one for $4 a$ to $8 a$ and one for $3 a$ to $9 a$, allowing, in each case, equal time separation from the source and sink. As can be seen, the values obtained are fully consistent, and, therefore, in what follows, we fix the fitting range to be the longer one. In all cases, we check that the final results are consistent with those extracted using the range $4 a$ to $8 a$.

\section{B. Simulation details}

The input parameters of the calculation, namely, $\beta, L / a$, and $a \mu$ are summarized in Table I. The lattice spacing $a$ is determined from the nucleon mass, and the reader is referred to Refs. [6,17] for more details. Here, we present only the final values, which are

$$
\begin{gathered}
a_{\beta=3.9}=0.089(1)(5), \quad a_{\beta=4.05}=0.070(1)(4), \\
a_{\beta=4.2}=0.056(2)(3),
\end{gathered}
$$

where the first error is statistical and the second systematic. The systematic error provides a measure of the uncertainty regarding the chiral extrapolation. It is estimated by comparing the value obtained using the $\mathcal{O}\left(p^{3}\right)$ and $\mathcal{O}\left(p^{4}\right)$ heavy baryon chiral perturbation theory. More details can be found in Ref. [25]. The pion mass values, spanning a range from 260 to $470 \mathrm{MeV}$, are taken from Ref. [37]. At $m_{\pi} \approx$ $300 \mathrm{MeV}$ and $\beta=3.9$, we have simulations for lattices of spatial size $L=2.1 \mathrm{fm}$ and $L=2.8 \mathrm{fm}$, allowing to investigate finite size effects. Finite lattice spacing effects are studied using three sets of results at $\beta=3.9, \beta=4.05$, and $\beta=4.2$ for the lowest and largest pion masses available in this work. These sets of gauge ensembles allow us to estimate all the systematic errors in order to produce reliable predictions for the nucleon one-derivative GFFs.

\section{Renormalization}

We determine the renormalization constants for the onederivative operators nonperturbatively, in the RI'-MOM scheme [38]. We employ a momentum source [39] and perform a perturbative subtraction of $\mathcal{O}\left(a^{2}\right)$ terms $[38,40]$.

TABLE I. Input parameters $(\beta, L, a \mu)$ of our lattice calculation and corresponding lattice spacing $(a)$ and pion mass $\left(m_{\pi}\right)$.

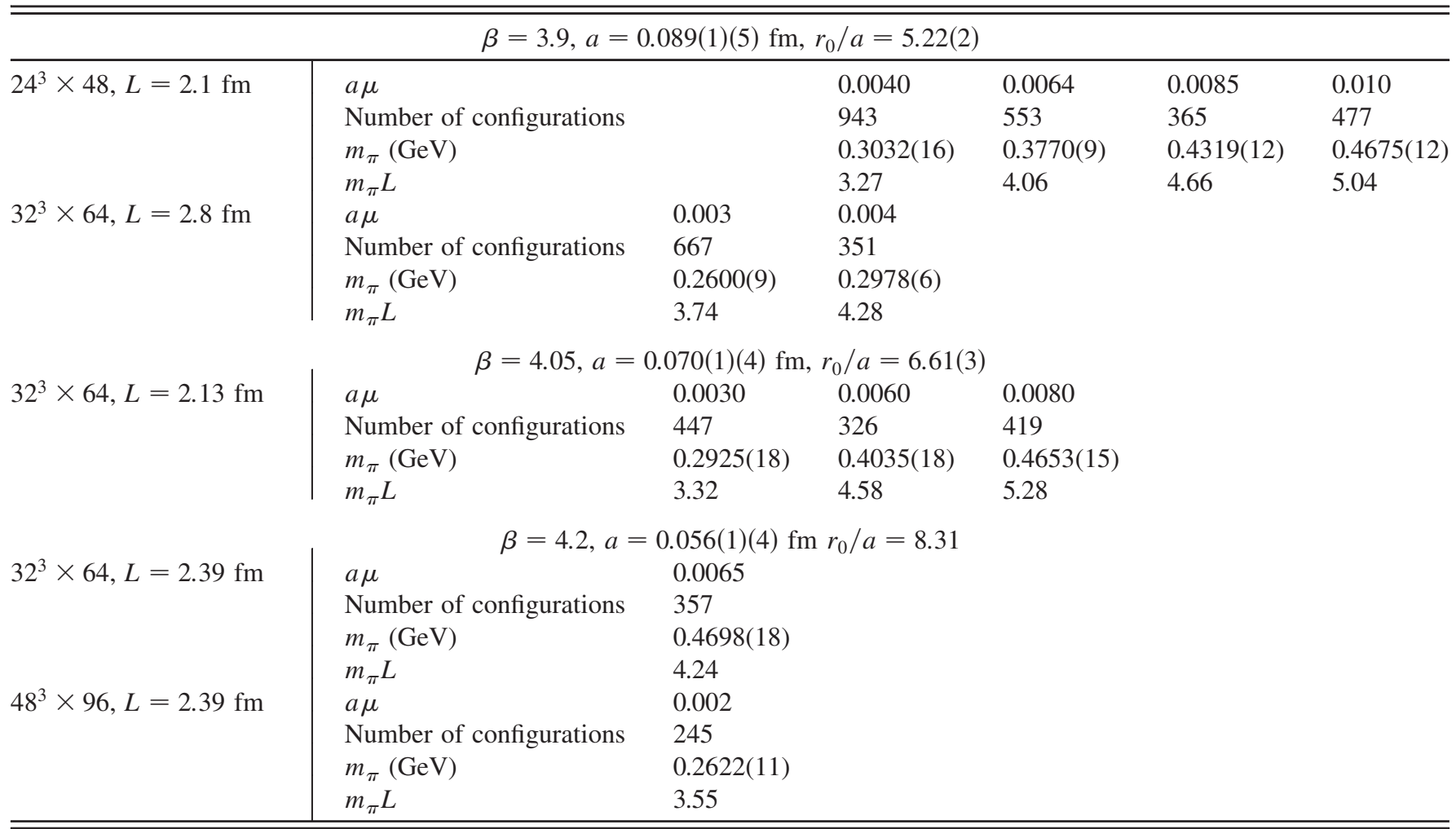


This subtracts the leading cutoff effects, yielding only a very weak dependence of the renormalization factors on $(a p)^{2}$, for which the $(a p)^{2} \rightarrow 0$ limit can be reliably taken. It was also shown with high accuracy that the quark mass dependence is negligible for the aforementioned operators. Converting into the $\overline{\mathrm{MS}}$ scheme at a scale $\mu=2 \mathrm{GeV}$, we find the values

$$
\begin{array}{llrl}
Z_{V}^{\mu=\nu}=0.970(26), & 1.033(14), & 1.097(6) ; \\
Z_{V}^{\mu \neq \nu}=1.061(29), & 1.131(18), & 1.122(10) ; \\
Z_{A}^{\mu \neq \nu}=1.076(1), & 1.136(0), & 1.165(10),
\end{array}
$$

at $\beta=3.9,4.05$, and 4.2 , respectively. These are the values that we use in this work to renormalize the lattice matrix element.

\section{LATTICE RESULTS}

In this section, we present our results on the nucleon generalized form factors $A_{20}\left(Q^{2}\right), B_{20}\left(Q^{2}\right), C_{20}\left(Q^{2}\right)$ and $\tilde{A}_{20}\left(Q^{2}\right), \tilde{B}_{20}\left(Q^{2}\right)$. We examine their dependence on the lattice volume and spacing, as well as on the pion mass. We also compare with recent results from other collaborations. In particular, we discuss lattice artifacts for the results on the isovector combination for the renormalized nucleon matrix element of the one-derivative operators

$$
\bar{u} \gamma_{\{\mu} \stackrel{\leftrightarrow}{D}_{\nu\}} u-\bar{d} \gamma_{\{\mu} \stackrel{\leftrightarrow}{D}_{\nu\}} d, \quad \bar{u} \gamma_{5} \gamma_{\{\mu} \stackrel{\leftrightarrow}{D}_{\nu\}} u-\bar{d} \gamma_{5} \gamma_{\{\mu} \stackrel{\leftrightarrow}{D}_{\nu\}} d
$$

in the $\overline{\mathrm{MS}}$ scheme at a scale $\mu=2 \mathrm{GeV}$. All the errors shown are statistical and are computed using the jackknife approach. To convert the momentum transfer to physical units, we use the lattice spacing as determined from the nucleon mass. Any systematic error in the determination of the lattice spacing affects only the value of $Q^{2}$ and has not been included in the analysis.

In order to obtain some estimates on the spin content of the nucleon, we also analyze the isoscalar parts of the spin-independent and helicity quark distributions, which, however, neglect the disconnected contributions.

The GFFs $A_{20}\left(Q^{2}=0\right)$ and $\tilde{A}_{20}\left(Q^{2}=0\right)$ are computed directly from the matrix elements, whereas $B_{20}\left(Q^{2}=0\right)$, $C_{20}\left(Q^{2}=0\right)$, and $\tilde{B}_{20}\left(Q^{2}=0\right)$ are obtained by linearly extrapolating the $Q^{2} \neq 0$ data. $C_{20}$ is consistent with zero within error bars for all momentum transfers.

\section{A. Finite volume effects}

In order to assess volume effects, we compare in Fig. 4 the results on the moments $\langle x\rangle_{u-d}$ and $\langle x\rangle_{\Delta u-\Delta d}$ computed on different lattice sizes as a function of $m_{\pi}^{2}$. As already mentioned, both of these quantities are directly obtained at $Q^{2}=0$ and require no assumption on their $Q^{2}$ dependence. Alongside our results, we also show results using $N_{F}=2$ clover fermions [41] (preliminary), $N_{F}=$ $2+1$ domain wall fermions (DWFs) [42], and domain

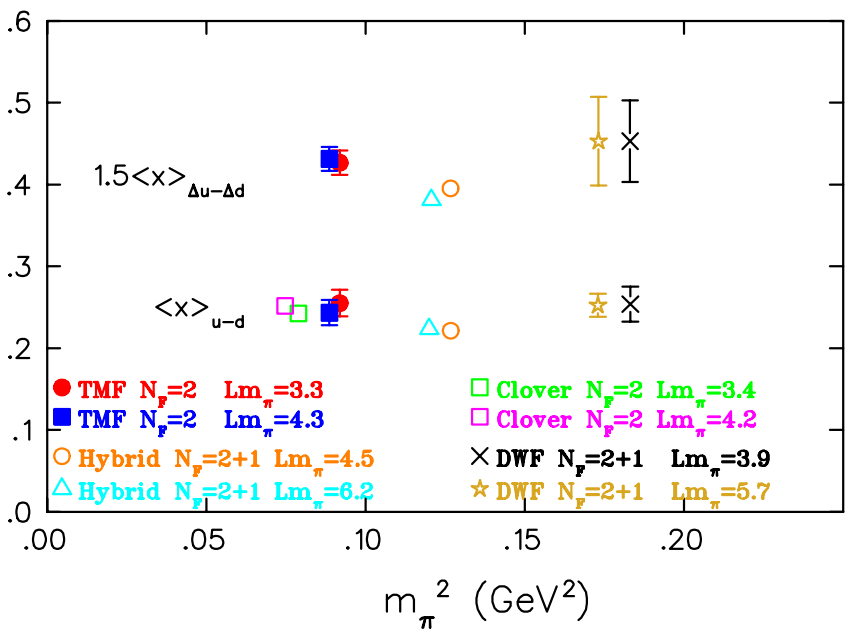

FIG. 4 (color online). $\langle x\rangle_{u-d}$ and $\frac{3}{2}\langle x\rangle_{\Delta u-\Delta d}$ using twisted mass fermions (this work), $N_{F}=2$ clover fermions [41] (preliminary), hybrids [43], and DWFs [42].

wall valence quarks on an $N_{F}=2+1$ staggered sea (hybrid) [43].

The results shown in Fig. 4 using twisted mass fermions correspond to a pion mass of about $300 \mathrm{MeV}$ and are computed on lattices of spatial $L$ with $L m_{\pi}=3.3$ and $L m_{\pi}=4.3$. As can be seen, the results on these two lattices for both $\langle x\rangle_{u-d}$ and $\langle x\rangle_{\Delta u-\Delta d}$ are consistent. The LHPC, using a hybrid approach and $m_{\pi} \sim 350 \mathrm{MeV}$, has very accurate results at two lattices with $L m_{\pi}=4.5$ and $L m_{\pi}=6.2$. No volume effects are seen for both vector and axial-vector first moments. The QCDSF Collaboration has preliminary results for $\langle x\rangle_{u-d}$, using clover fermions for $m_{\pi} \sim 270 \mathrm{MeV}$ with $L m_{\pi}=3.4$ and $L m_{\pi}=4.2$, which are consistent. Finally the RBC-UKQCD results with domain wall fermions with $L m_{\pi}=3.9$ and $L m_{\pi}=5.7$ show no volume effects for both $\langle x\rangle_{u-d}$ and $\langle x\rangle_{\Delta u-\Delta d}$ [42]. The conclusion that we draw from this comparison is that finite volume effects on $\langle x\rangle_{u-d}$ and $\langle x\rangle_{\Delta u-\Delta d}$ are insignificant at our current statistical precision for lattices that satisfy $L m_{\pi}>3.3$.

In Figs. 5, we compare results on the GFFs $A_{20}\left(Q^{2}\right)$, $B_{20}\left(Q^{2}\right), \tilde{A}_{20}\left(Q^{2}\right)$, and $\tilde{B}_{20}\left(Q^{2}\right)$ using twisted mass fermions for $m_{\pi} \sim 300 \mathrm{MeV}$ for our two spatial lattice sizes of $L=2.1 \mathrm{fm}$ and $L=2.8 \mathrm{fm}$. The lines shown are linear fits to the $Q^{2}$ dependence. As can be seen, for both $A_{20}\left(Q^{2}\right)$ and $\tilde{A}_{20}\left(Q^{2}\right)$, one cannot ascertain any volume dependence. For $B_{20}\left(Q^{2}\right)$ and $\tilde{B}_{20}\left(Q^{2}\right)$, the statistical errors are larger, and the linear fits show a larger spread with the change in the spatial volume. However, given the large statistical uncertainties, it is difficult to quantify any volume dependence.

\section{B. Cutoff effects}

In order to examine cutoff effects, we compare in Fig. 6 our results obtained at the lowest and highest pion masses 

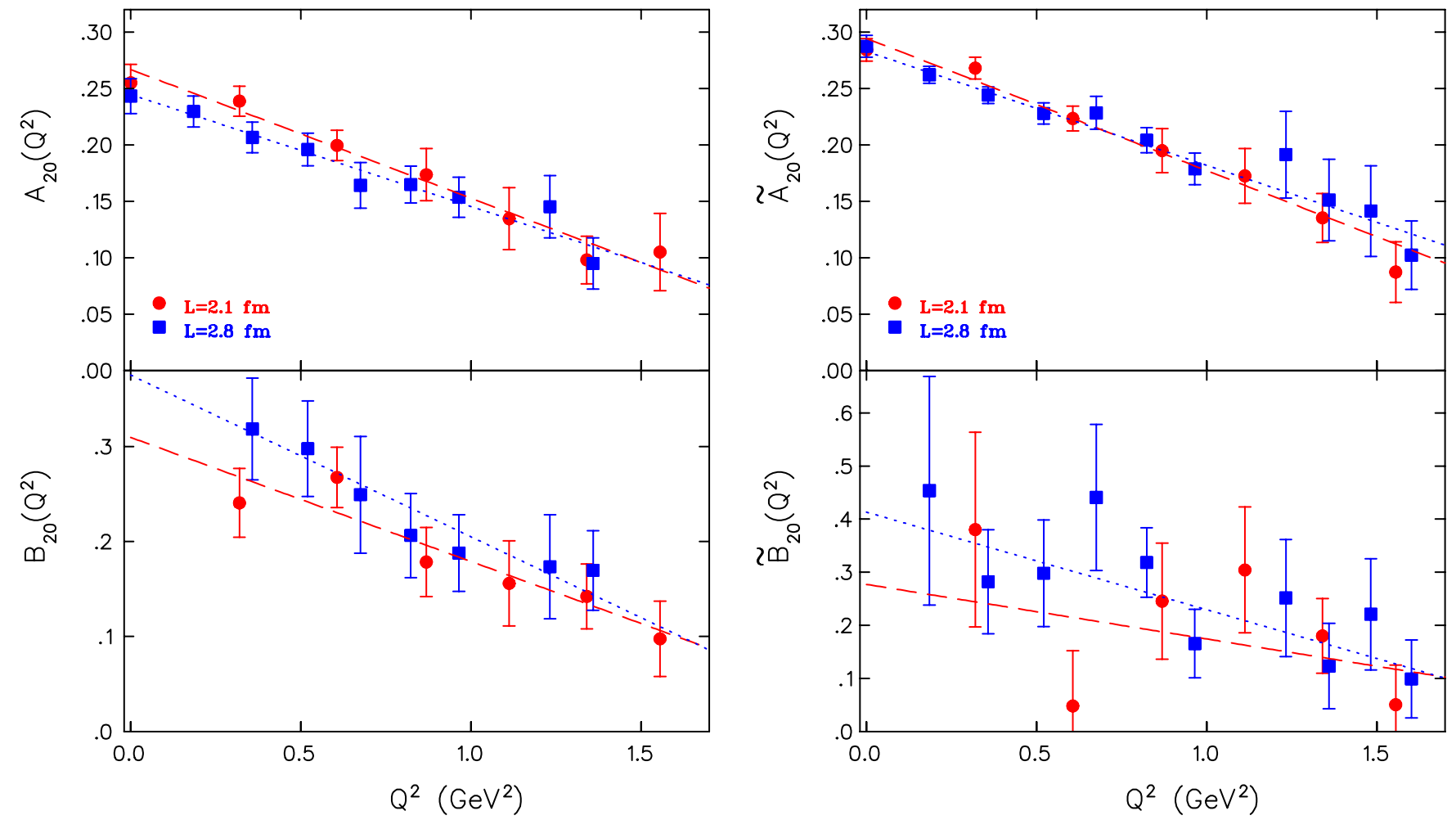

FIG. 5 (color online). Left panel: the isovector GFFs $A_{20}$ and $B_{20}$ for pion mass $\sim 300 \mathrm{MeV}$ for a lattice of spatial length $L=2.1$ fm and $L=2.8 \mathrm{fm}$. Right panel: the axial-vector GFFs $\tilde{A}_{20}$ and $\tilde{B}_{20}$ for $L=2.1 \mathrm{fm}$ and $L=2.8 \mathrm{fm}$.
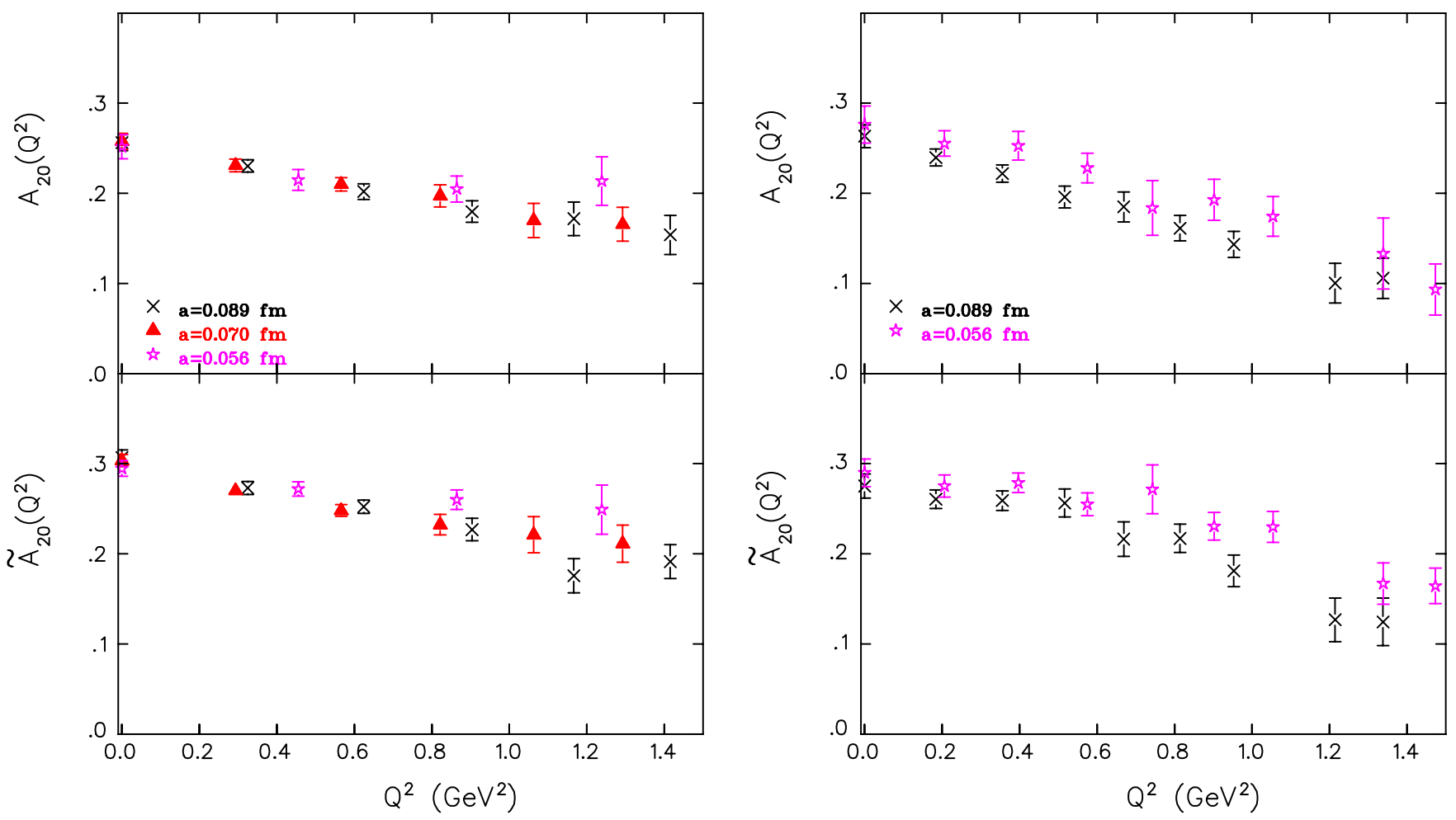

FIG. 6 (color online). Left panel: the GFFs $A_{20}$ and $\tilde{A}_{20}$ for our three lattice spacings at $m_{\pi} \sim 470 \mathrm{MeV}$. Right panel: $A_{20}$ and $\tilde{A}_{20}$ for $a=0.089 \mathrm{fm}$ and $a=0.056 \mathrm{fm}$ at $m_{\pi} \sim 260 \mathrm{MeV}$. 


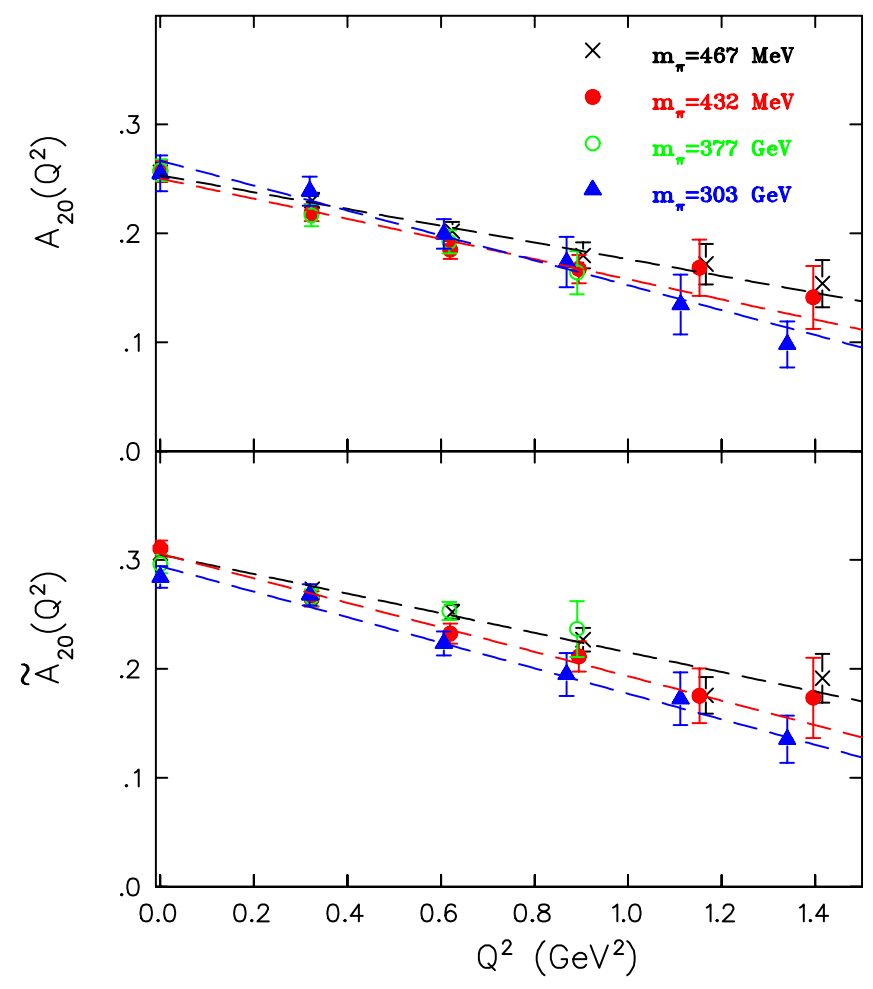

FIG. 7 (color online). Pion mass dependence of $A_{20}\left(Q^{2}\right)$ and $\tilde{A}_{20}\left(Q^{2}\right)$, computed at $\beta=3.9$ and using a lattice size of $24^{3} \times 48$.

that we have considered in this work, namely, $m_{\pi} \sim$ $260 \mathrm{MeV}$ and $m_{\pi} \sim 470 \mathrm{MeV}$. We show results on the quantities $A_{20}\left(Q^{2}\right)$ and $\tilde{A}_{20}\left(Q^{2}\right)$, since these have smaller statistical errors than $B_{20}\left(Q^{2}\right)$ and $\tilde{B}_{20}\left(Q^{2}\right)$. For the heavier mass, where we have results at all three lattice spacings, there is no visible dependence on the lattice spacing, especially at low $Q^{2}$ values. For the lightest pion mass of $m_{\pi}=260 \mathrm{MeV}$, we have results at the largest and smallest lattice spacings. The results are in good agreement, although some deviations are seen at larger $Q^{2}$ values. Thus, within our current statistical errors, one may conclude that no significant cutoff effects are observed.

\section{Quark mass dependence}

The mass dependence of $A_{20}$ and $\tilde{A}_{20}$ is shown in Fig. 7. Although the dependence on the mass is weak in the range of pion masses spanned, the tendency is for the values of the GFFs to decrease with decreasing pion mass. There is also a tendency for an increase in the slope for both $A_{20}$ and $\tilde{A}_{20}$ as the pion mass decreases.

\section{Comparison with other discretization schemes}

In order to compare lattice data using different discretization schemes, one would have to first extrapolate to the continuum limit. However, given that the cutoff effects are small for lattice spacings of about $0.1 \mathrm{fm}$, lattice results for different values of $a$ using a number of improved discretizations can be directly compared. In Fig. 8, we show results on the spin-independent and helicity moments using twisted mass fermions in the hybrid approach obtained by LHPC [43], with $N_{F}=2$ clover fermions by the QCDSF Collaboration [41] (preliminary results), and with DWFs by the RBC-UKQCD Collaborations [42]. There is good agreement among the lattice results. The very accurate results obtained using a hybrid action of domain wall valence and $N_{F}=2+1$ staggered fermions [43] tend to be lower, compared to the other data. One difference
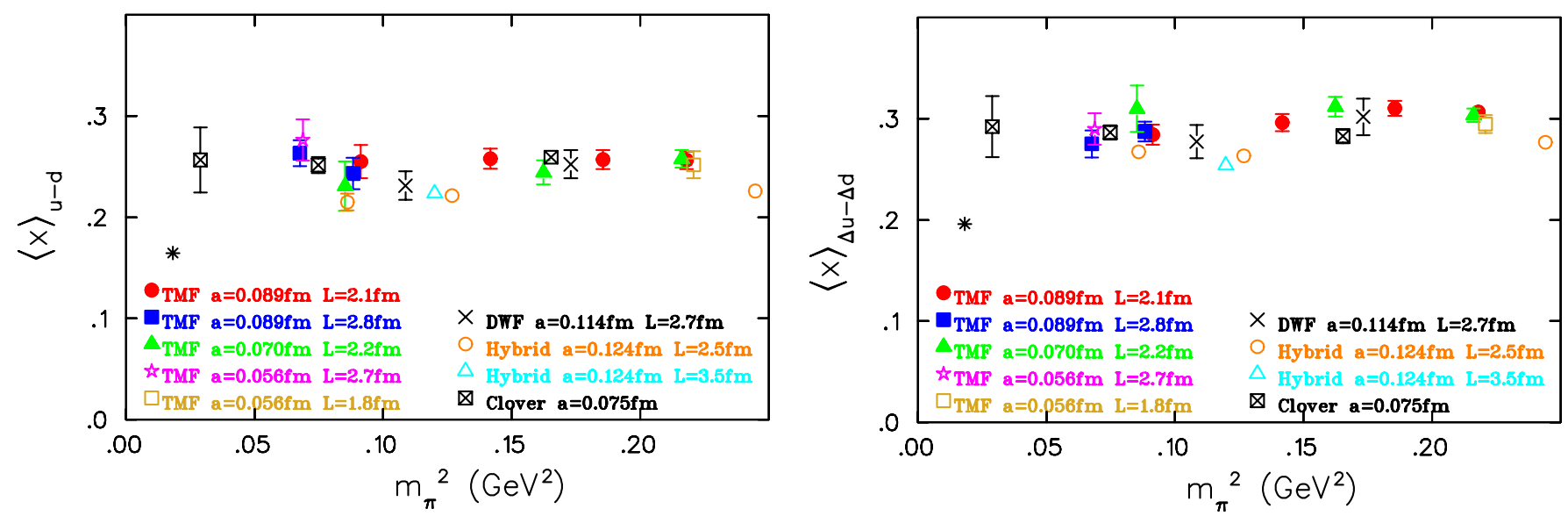

FIG. 8 (color online). Lattice data on $\langle x\rangle_{u-d}$ and $\langle x\rangle_{\Delta u-\Delta d}$, using (i) $N_{F}=2$ twisted mass fermions: $a=0.089$ fm: filled red circles for $L=2.1 \mathrm{fm}$ and filled blue squares for $L=2.8 \mathrm{fm}, a=0.070 \mathrm{fm}$ : filled green triangles for $L=2.2 \mathrm{fm}, a=0.056 \mathrm{fm}$ : purple stars for $L=2.7 \mathrm{fm}$, and open yellow squares for $L=1.8 \mathrm{fm}$; (ii) $N_{F}=2+1$ DWFs [42]: crosses for $a=0.114 \mathrm{fm}$ and $L=2.7 \mathrm{fm}$; (iii) $N_{F}=2+1$ using DWFs for the valence quarks on a staggered sea [43]: $a=0.124$ fm: open orange circles for $L=2.5 \mathrm{fm}$ and open cyan triangles for $L=3.5 \mathrm{fm}$; (iv) $N_{F}=2$ clovers: $a=0.075 \mathrm{fm}$ [41] (preliminary results). The physical point, shown by the asterisk, is from Ref. [50] for the unpolarized and from Refs. [51,52] for the polarized first moment. 
between them and the other results presented is that they are perturbatively renormalized. It was shown in Ref. [42] that perturbative renormalization can lead to lower values. The spread in the values of the lattice results is shown to be reduced by taking a renormalization-free ratio, leading to a better agreement among lattice data with $L m_{\pi}>4$ [44]. In particular, constructing a renormalization-free ratio brought the hybrid data in agreement with our results using twisted mass fermions and those using clover fermions by QCDSF. Lattice values for $\langle x\rangle_{u-d}=A_{20}\left(Q^{2}=0\right)$, although compatible, are higher from the phenomenological value $\left\langle x_{u-d}\right\rangle \sim 0.16$. The very recent preliminary result by QCDSF [41] at $m_{\pi} \sim 170 \mathrm{MeV}$ remains higher than the experiment and highlights the need to understand such deviations. A similar conclusion holds for the helicity moment.

In Figs. 9, we compare our results for the GFFs with pion mass $300 \mathrm{MeV}$ with those obtained using a hybrid action by LHPC and clover fermions by QCDSF, with pion masses of 355 and $350 \mathrm{MeV}$, respectively. The results show an overall agreement, with the data of LHPC somewhat lower than the other two sets. Once more, the fact that both our results and those of QCDSF are renormalized nonperturbatively, while those of LHPC are renormalized perturbatively, might explain this difference. Moreover, in our determination of the renormalization constants, we have subtracted $\mathcal{O}\left(a^{2}\right)$ terms perturbatively to reduce lattice artifacts [38].

\section{CHIRAL PERTURBATION THEORY}

In order to make a direct comparison with the experiment, we need to extrapolate to the physical point. Our lattice results are obtained using pion masses in the range of 260 to $470 \mathrm{MeV}$. We expect chiral expansions for baryonic quantities to hold below about $300 \mathrm{MeV}$. Therefore, our study here is to examine the overall qualitative behavior. We only perform a chiral extrapolation of GFFs at $Q^{2}=0$. We first perform this extrapolation using our lattice results directly, since, as we have discussed in
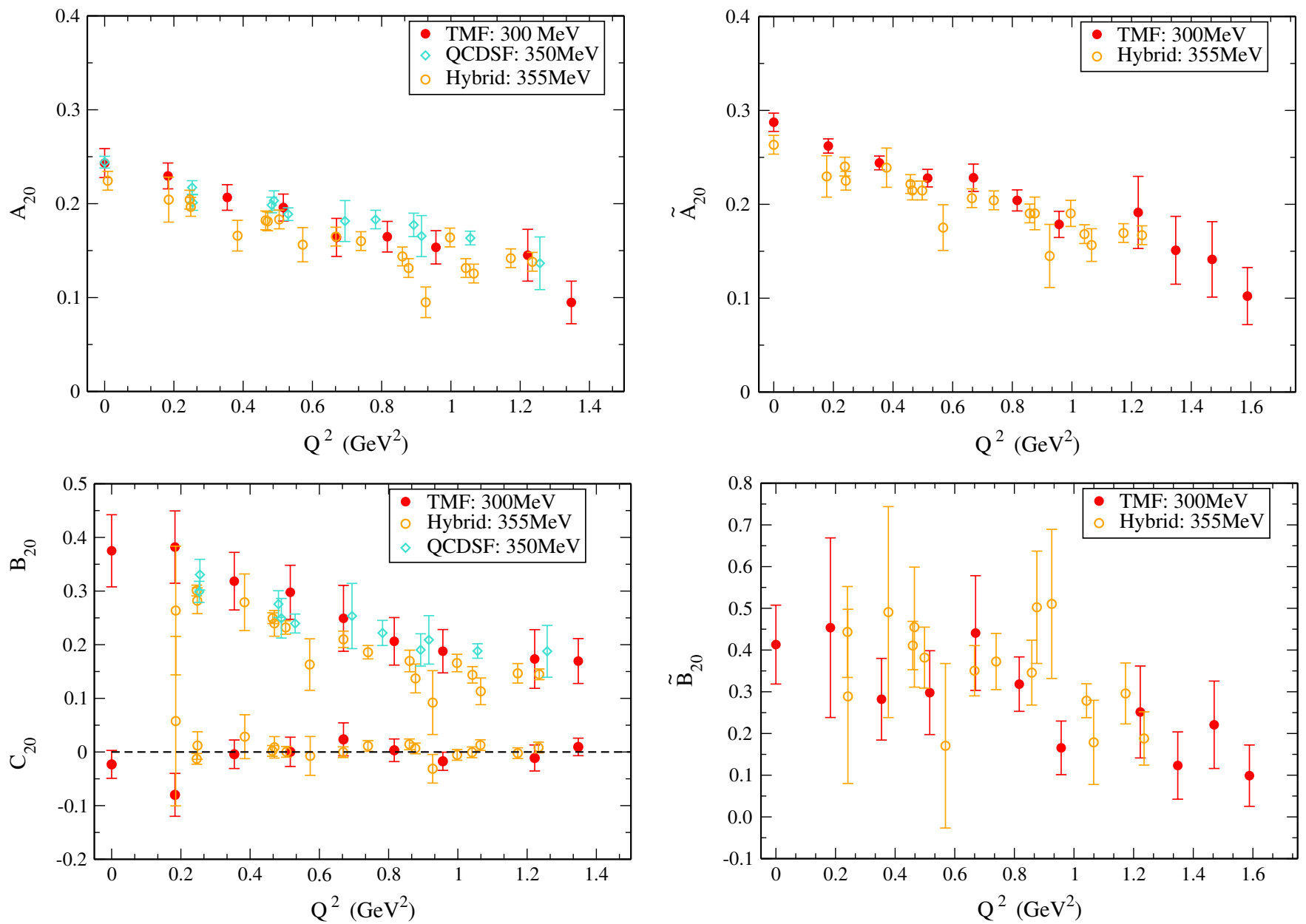

FIG. 9 (color online). Comparison of twisted mass results for $A_{20}\left(Q^{2}\right), B_{20}\left(Q^{2}\right)$, and $C_{20}\left(Q^{2}\right)\left(\right.$ left panel) and $\tilde{A}_{20}\left(Q^{2}\right)$ and $\tilde{B}_{20}\left(Q^{2}\right)$ (right panel) at pion mass $300 \mathrm{MeV}$ with those obtained using a hybrid action at $m_{\pi}=355 \mathrm{MeV}$ and $N_{F}=2$ clover fermions at $m_{\pi}=350 \mathrm{MeV}$. Hybrid results are from Ref. [43], and the results using clover fermions are from Ref. [53]. 
the previous section, cutoff effects are small. In the next section, we will perform a continuum extrapolation and verify that, indeed, the values we find at the physical point are compatible.

Within heavy baryon chiral perturbation theory (HB $\chi \mathrm{PT}$ ) [45,46], the expressions for the $m_{\pi}$ dependence of $A_{20}$ and $\tilde{A}_{20}$ are given by

$$
\langle x\rangle_{u-d}=C\left[1-\frac{3 g_{A}^{2}+1}{\left(4 \pi f_{\pi}\right)^{2}} m_{\pi}^{2} \ln \frac{m_{\pi}^{2}}{\lambda^{2}}\right]+\frac{c_{8}\left(\lambda^{2}\right) m_{\pi}^{2}}{\left(4 \pi f_{\pi}\right)^{2}},
$$

$$
\langle x\rangle_{\Delta u-\Delta d}=\tilde{C}\left[1-\frac{2 g_{A}^{2}+1}{\left(4 \pi f_{\pi}\right)^{2}} m_{\pi}^{2} \ln \frac{m_{\pi}^{2}}{\lambda^{2}}\right]+\frac{\tilde{c}_{8}\left(\lambda^{2}\right) m_{\pi}^{2}}{\left(4 \pi f_{\pi}\right)^{2}},
$$

where we take $\lambda^{2}=1 \mathrm{GeV}^{2}$. The best fit is shown in Fig. 10, where the width of the band is computed through a superjackknife analysis [43]. As can be seen, the fits yield a value higher than the experiment for both observables. LHPC carried out a combined chiral fit using the $\mathcal{O}\left(p^{2}\right)$ covariant baryon chiral perturbation theory $(\mathrm{CB} \chi \mathrm{PT})$ [47] to $A_{20}, B_{20}$, and $C_{20}$. The mass of the nucleon at the chiral
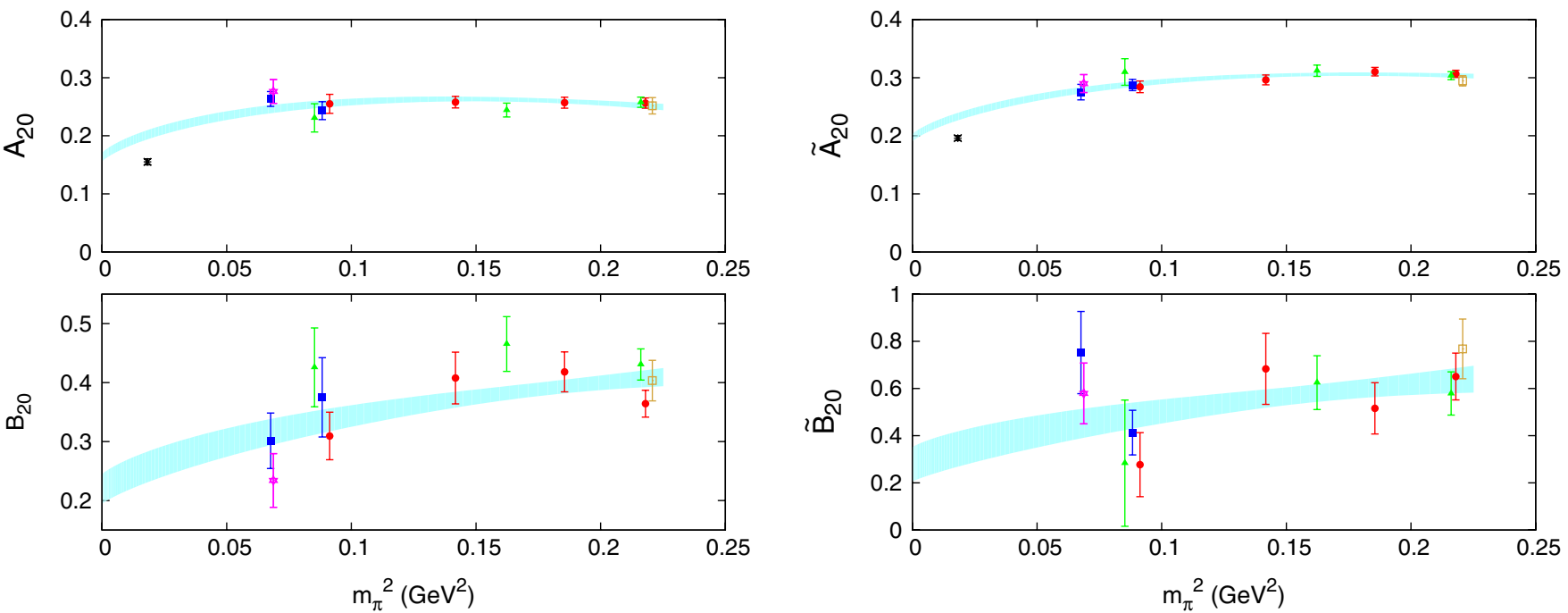

FIG. 10 (color online). Chiral extrapolation using $\mathrm{HB} \chi \mathrm{PT}$ for the isovector unpolarized and polarized first moments of the quark distributions. The physical point, shown by the asterisk, is from Ref. [50] for the unpolarized and from Refs. [51,52] for the polarized first moment.
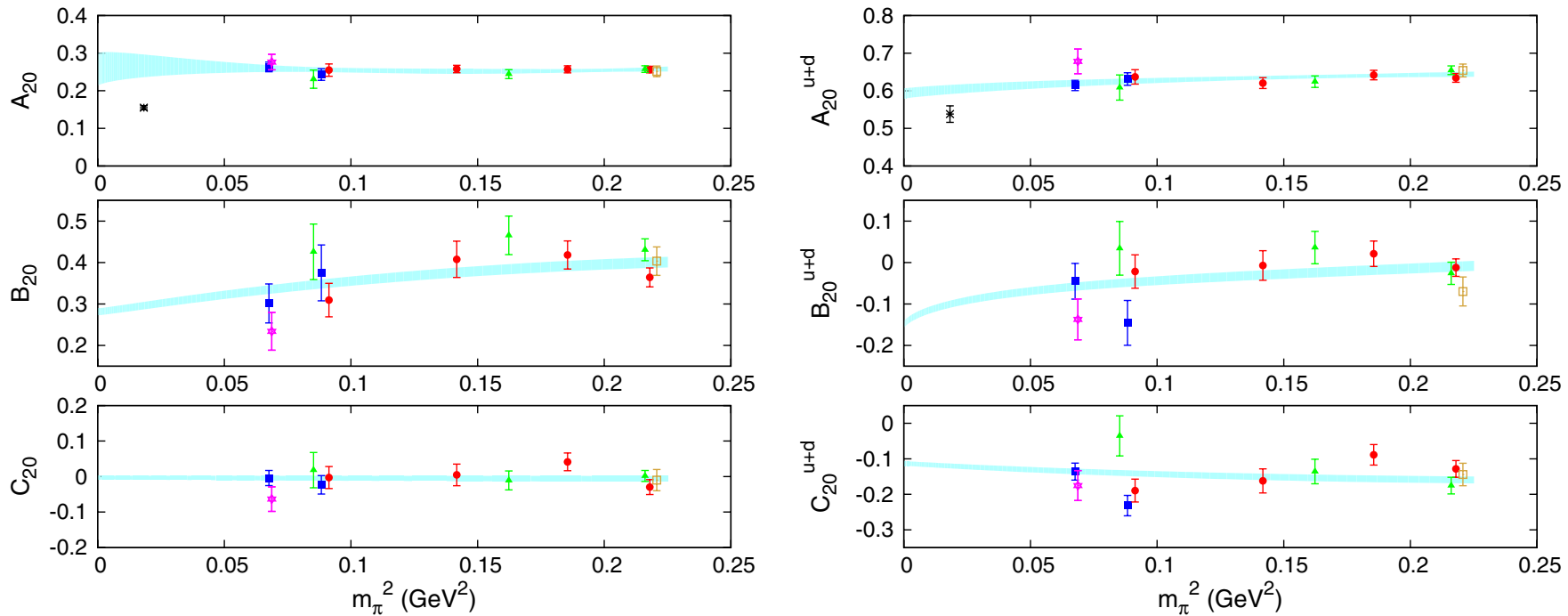

FIG. 11 (color online). Chiral extrapolation using $\mathrm{CB} \chi \mathrm{PT}$ for the isovector (left) and isoscalar (right) moments $A_{20}, B_{20}$, and $C_{20}$. The physical point, shown by the asterisk, is from Ref. [50] for the isovector and from Refs. [54,55] for the isoscalar. 
limit is used as input to the fits. They obtained a value for $A_{20}$ in agreement with the experiment [43]. In order to compare with their analysis, we also perform a combined fit to $A_{20}(0), B_{20}(0)$, and $C_{20}(0)$ within $\mathrm{CB} \chi \mathrm{PT}$ [47]. The $\mathrm{CB} \chi \mathrm{PT}$ fits are shown by the bands in Fig. 11. As can be seen, they also provide a good description to the lattice data, but, in the case of $A_{20}, \mathrm{CB} \chi \mathrm{PT}$ leads to an even higher value at the physical point. Therefore, the discrepancy between our lattice results and the experimental value is not resolved. In Appendix B, we collect the formulas used for the chiral extrapolations. The actual renormalized lattice data are tabulated in Tables III, IV, V, and VI of Appendix C for the isovector GFFs.

\section{CONTINUUM EXTRAPOLATION}

In order to study the continuum extrapolation, we use the simulations at our three lattice spacings at the smallest and largest pion masses. We first interpolate the GFFs at the three values of $\beta$ to a given value of the pseudoscalar mass in units of $r_{0}$. We take as reference pion masses the ones computed on the finest lattice and interpolate results at the other two $\beta$ values to these two reference masses.

As already mentioned, $\langle x\rangle_{u-d}=A_{20}(0)$ and $\langle x\rangle_{\Delta u-\Delta d}=$ $\tilde{A}_{20}(0)$ are calculated directly at $Q^{2}=0$, requiring no fits. We, therefore, choose these quantities to examine their dependence on the lattice spacing, since this choice avoids
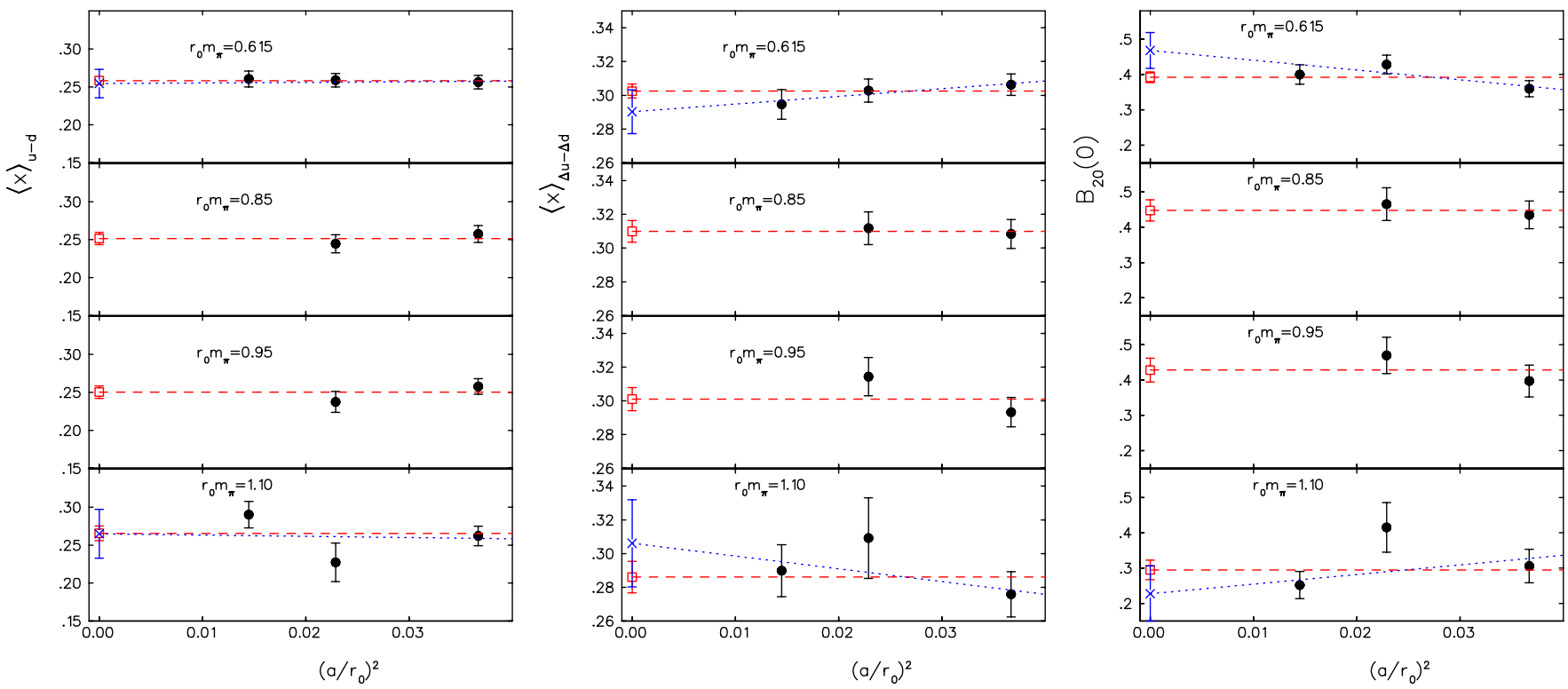

FIG. 12 (color online). $\langle x\rangle_{u-d},\langle x\rangle_{\Delta u-\Delta d}$, and $B_{20}(0)$ as a function of $\left(a / r_{0}\right)^{2}$. The dashed red line is the result of fitting to a constant; the dotted blue one is a linear fit.
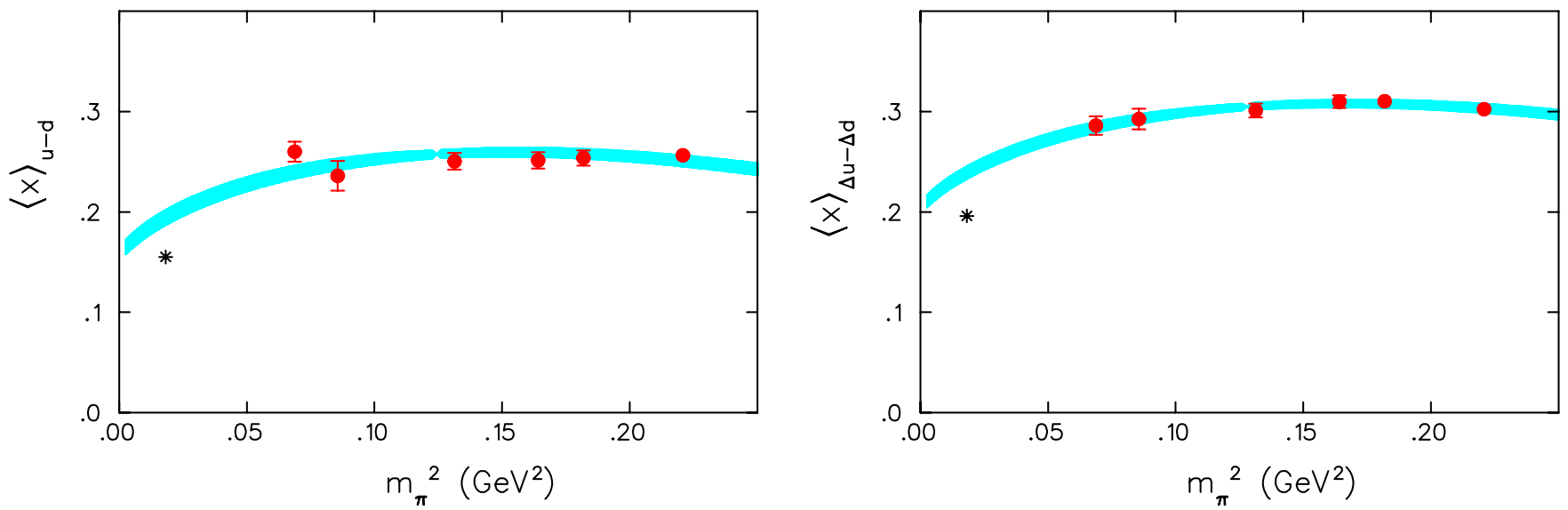

FIG. 13 (color online). $\langle x\rangle_{u-d}$ (left) and $\langle x\rangle_{\Delta u-\Delta d}$ (right) extrapolated to $a=0$ as a function of $m_{\pi}$. The blue band is the chiral fit using $\mathrm{HB} \chi \mathrm{PT}$. The physical point, shown by the asterisk, is from Ref. [50] for the unpolarized and from Refs. [51,52] for the polarized first moment. 
any systematic errors, due to the extrapolation to $Q^{2}=0$, which would require the adaptation of an ansatz for the $Q^{2}$ dependence.

Having determined the values at a given reference pion mass, we perform a fit to these data using the form $y(a)=$ $y(0)+c\left(a / r_{0}\right)^{2}$. The resulting fits are shown in Fig. 12 . Setting $c=0$, we obtained the constant line also shown in the figure. As can be seen, for both large and small pion masses, allowing a nonzero slope yields a value in the continuum limit that is in agreement with that obtained using a constant fit. This analysis shows that finite $a$ effects are small for both large and small pion masses, and extrapolation to the continuum limit using a constant fit is acceptable. For the intermediate pion masses, we, therefore, obtained the values in the continuum by fitting our data at $\beta=3.9$ and $\beta=4.05$ to a constant. For comparison, we also perform a similar analysis for $B_{20}(0)$, which requires fitting the $Q^{2}$ dependence. The qualitative behavior is similar to that observed for $A_{20}(0)$ and $B_{20}(0)$.

Having results at the continuum limit, we perform a chiral fit using $\mathrm{HB} \chi \mathrm{PT}$. The resulting curves are shown in Fig. 13 and still produce a value at the physical point that is higher than the experimental value. In fact, the value obtained at the physical point for both vector and axialvector moments is in agreement to the one extracted using the raw lattice data. This provides an a posteriori justification of using continuum chiral perturbation theory directly on the lattice data obtained at our three lattice spacings to perform the extrapolation to the physical point in the previous section.

\section{PROTON SPIN}

In order to extract information on the spin content of the nucleon, one needs to evaluate the isoscalar moments $A_{20}^{u+d}$ and $B_{20}^{u+d}$, since the total spin of a quark in the nucleon is given by [10]

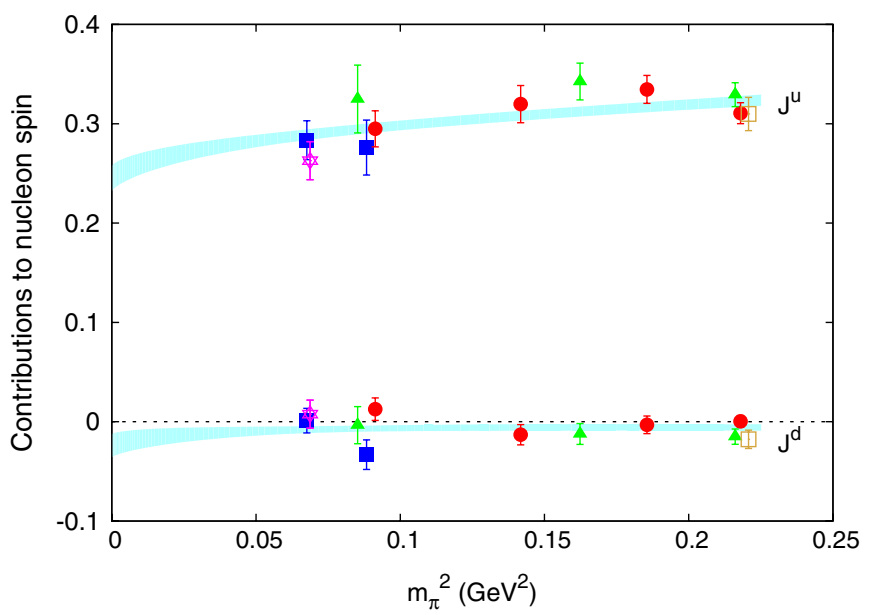

$$
J^{q}=\frac{1}{2}\left(A_{20}^{q}(0)+B_{20}^{q}(0)\right) .
$$

The total spin can be further decomposed into its orbital angular momentum $L^{q}$ and its spin component $\Delta \Sigma^{q}$ as

$$
J^{q}=\frac{1}{2} \Delta \Sigma q+L^{q} .
$$

The spin carried by the $u$ and $d$ quarks is determined using $\Delta \Sigma^{u+d}=\tilde{A}_{10}^{u+d}$. In order to evaluate the isoscalar quantities, one would need the disconnected contributions. These are notoriously difficult to calculate, and they are neglected in most current evaluations of GFFs. Under the assumption that these are small, we may extract the information on the nucleon spin.

In Fig. 11, we show our results for the isoscalar $A_{20}(0)^{u+d}, B_{20}(0)^{u+d}$, and $C_{20}(0)^{u+d}$. Since, as shown in the previous section, cutoff effects are small, we here perform a chiral extrapolation directly on the lattice data. Having both isoscalar and isovector quantities, we can extract the spin $J^{u}$ and $J^{d}$ carried by the $u$ and $d$ quarks. The results are shown in Fig. 14. We show the extrapolation using both $\mathrm{HB} \chi \mathrm{PT}$ and $\mathrm{CB} \chi \mathrm{PT}$, both of which have the same qualitative behavior. As can be seen, the contribution to the spin from the $d$ quark is much smaller than that of the $u$ quarks. These results are in qualitative agreement with the recent results obtained using a hybrid action [43]. In Fig. 15, we show separately the orbital angular momentum and spin carried by the $u$ and $d$ quarks. Both our results and those of LHPC [43] are in qualitative agreement, as far as the spin is concerned. For the orbital angular momentum, we obtain higher values for both $d$ and $u$ quarks (less negative). Thus, we obtain a total positive $L^{u+d}$, compared to a small negative value in the case of LHPC. After chiral extrapolation, the value obtained at the physical point is consistent with zero, in agreement with the value determined by LHPC. For the spin contribution $\Delta \Sigma^{u+d}$, our value is lower at the physical point, as compared to that obtained by LHPC. We summarize the values for the total spin, orbital angular

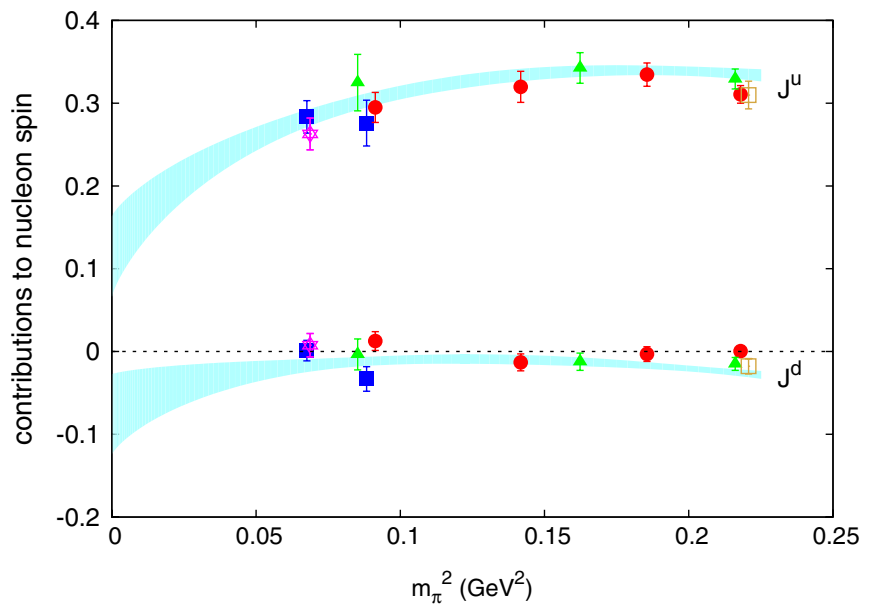

FIG. 14 (color online). Chiral extrapolation using $\mathrm{CB} \chi \mathrm{PT}$ (left) and $\mathrm{HB} \chi \mathrm{PT}$ (right) for the total spin carried by the $u$ and $d$ quarks. 

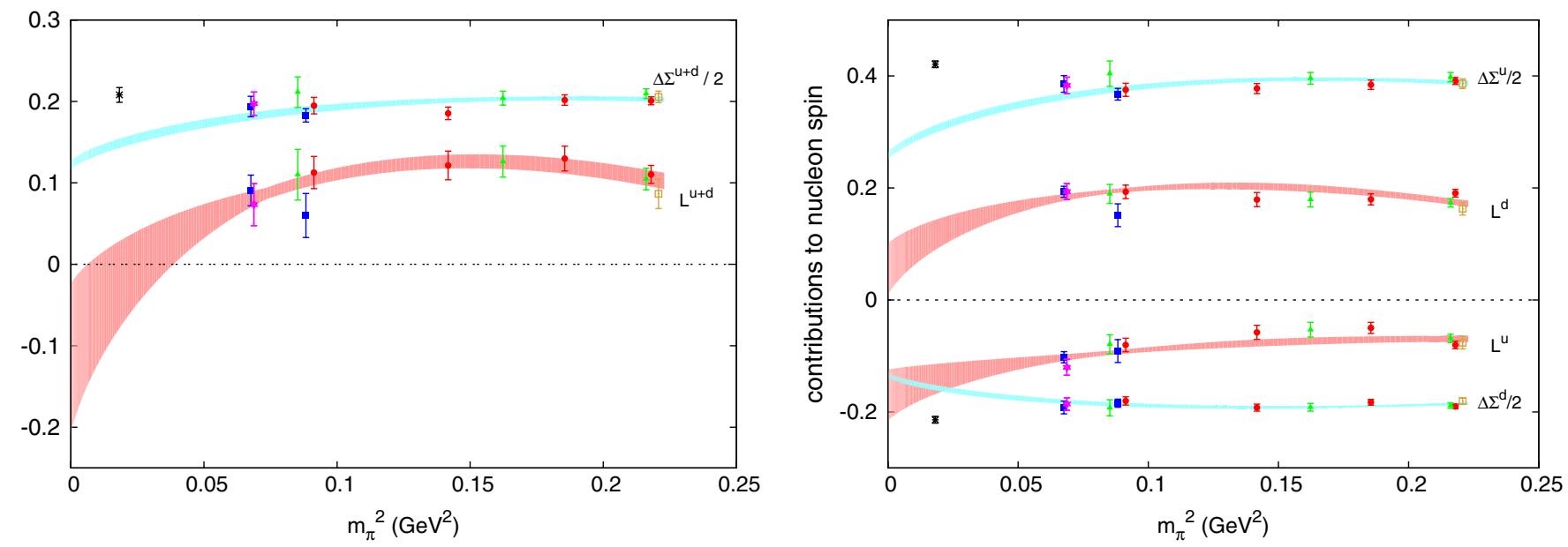

FIG. 15 (color online). Chiral extrapolation using HB $\chi \mathrm{PT}$ for the angular momentum and spin carried by the $u$ and $d$ quarks. The physical points, shown by the asterisks, are from the HERMES 2007 analysis [48].

TABLE II. Values of nucleon spin observables at the physical point, using $\mathrm{CB} \chi \mathrm{PT}$ and $\mathrm{HB} \chi \mathrm{PT}$, and from the experiment [48].

\begin{tabular}{lrrr}
\hline \hline & $\mathrm{CB} \chi \mathrm{PT}$ & $\mathrm{HB} \chi \mathrm{PT}$ & Experiment \\
\hline$J^{u-d}$ & & $0.236(14)$ & \\
$J^{u+d}$ & & $0.143(56)$ & \\
$J^{u}$ & $0.266(9)$ & $0.189(29)$ & \\
$J^{d}$ & $-0.015(8)$ & $-0.047(28)$ & \\
$\Delta \Sigma^{u-d} / 2$ & & $0.462(11)$ & \\
$\Delta \Sigma^{u+d} / 2$ & & $0.148(5)$ & $0.208(9)$ \\
$\Delta \Sigma^{u} / 2$ & $0.305(7)$ & $0.421(6)$ \\
$\Delta \Sigma^{d} / 2$ & & $-0.157(5)$ & $-0.214(6)$ \\
$L^{u-d}$ & & $-0.258(5)$ & \\
$L^{u+d}$ & & $-0.025(53)$ & \\
$L^{u}$ & & $-0.141(30)$ & \\
$L^{d}$ & & $0.116(27)$ & \\
\hline \hline
\end{tabular}

momentum, and spin in the proton at the physical point in Table II. We would like to stress that simulations with pion masses closer to the physical point would be needed to check the validity of the chiral extrapolations of these quantities. Such simulations are currently being performed.

\section{CONCLUSIONS}

We have performed an analysis on the generalized form factors, $A_{20}\left(Q^{2}\right), B_{20}\left(Q^{2}\right), C_{20}\left(Q^{2}\right), \tilde{A}_{20}\left(Q^{2}\right)$, and $\tilde{B}_{20}\left(Q^{2}\right)$, extracted from the nucleon matrix elements of the onederivative vector and axial-vector operators using two degenerate flavors of twisted mass fermions. Our results are nonperturbatively renormalized, and they are presented in the $\overline{\mathrm{MS}}$ scheme at a scale of $2 \mathrm{GeV}$. To investigate volume and cutoff effects, we have used the isovector combinations, which can be calculated without the necessity to evaluate disconnected contributions. Our main conclusion regarding cutoff effects is that they are small within the current accuracy of about $5-10 \%$ and for lattice spacings smaller than $0.1 \mathrm{fm}$. Similarly, no systematic volume effects are seen. Given the small cutoff effects, one can compare lattice results directly using different discretization schemes. The comparison of the results using $N_{F}=2$ twisted mass fermions with the results obtained using $N_{F}=2$ clover fermions by the QCDSF [41] shows agreement. Both the results of this work as well as those by QCDSF are nonperturbatively renormalized. We also compared our results with $N_{F}=2+1$ domain wall fermions [42]. Again, there is agreement without any indication of any systematic effect from including a dynamical strange quark. Our results at three values of the lattice spacing allow for a continuum extrapolation. By interpolating results to a reference mass in units of $r_{0}$ and performing a linear extrapolation in $a^{2}$, it was shown that the values obtained are consistent with those obtained with a constant extrapolation. This has been verified for both the heaviest and lightest masses used in this work. Furthermore, if one performs chiral fits to the extrapolated continuum results, one finds a value at the physical point consistent with the one obtained using directly the lattice data at finite lattice spacing. This provides a consistency check that cutoff effects for a lattice spacing less than $0.1 \mathrm{fm}$ are smaller than our current statistical errors.

Having established that both volume and cutoff effects are small for the isovector quantities for which only connected contributions are needed, we analyze the corresponding isoscalar quantities using directly our lattice data. Of particular interest here is the spin content of the nucleon. The disconnected contributions to the isoscalar quantities are not included. We find that the spin carried by the $d$ quark is almost zero, whereas the $u$ quarks carry about $50 \%$ of the nucleon's spin. This result is consistent with other lattice calculations [43].

For the chiral extrapolations of these quantities, we use $\mathrm{HB} \chi \mathrm{PT}$ and $\mathrm{CB} \chi \mathrm{PT}$. In both cases, our results on the momentum fraction and helicity moment at the physical point are higher than their experimental value. Such 
discrepancies are also observed in the case of the nucleon axial charge, and they need to be further investigated.

\section{ACKNOWLEDGMENTS}

We would like to thank all members of ETMC for a very constructive and enjoyable collaboration and for the many fruitful discussions that took place during the development of this work. Numerical calculations have used HPC resources from GENCI (IDRIS and CINES) Grants No. 2009-052271 and No. CC-IN2P3, as well as from the John von Neumann Institute for Computing on the Juropa and Jugene systems at the research center in Jülich. We thank the staff members for their kind and sustained support. M. Papinutto acknowledges financial support by the Marie Curie European Reintegration Grant of the 7th European Community Framework Programme under Contract No. PERG05-GA-2009-249309. This work is supported in part by the DFG Sonderforschungsbereich/ Transregio SFB/TR9 and by funding received from the Cyprus Research Promotion Foundation under Contracts No. EPYAN/0506/08, No. KY $-\Gamma / 0907 / 11 /$, and

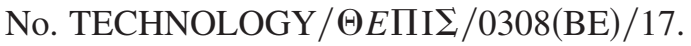

\section{APPENDIX A: EXPRESSIONS FOR THE EXTRACTION OF GFFs FROM LATTICE MEASUREMENTS}

We collect here the expressions relating the plateau values to the GFFs $A_{20}, B_{20}, C_{20}$ and $\tilde{A}_{20}, \tilde{B}_{20}$. The index $V(A)$ refers to the vector (axial-vector) one-derivative operator. All relations are given in Euclidean space.

Vector:

$$
\begin{aligned}
\Pi_{V}^{00}\left(\Gamma^{0}, \vec{q}\right)= & A_{20} C\left(-\frac{3 E_{N}}{8}-\frac{E_{N}^{2}}{4 m_{N}}-\frac{m_{N}}{8}\right) \\
& +B_{20} C\left(-\frac{E_{N}}{8}+\frac{E_{N}^{3}}{8 m_{N}^{2}}+\frac{E_{N}^{2}}{16 m_{N}}-\frac{m_{N}}{16}\right) \\
& +C_{20} C\left(\frac{E_{N}}{2}-\frac{E_{N}^{3}}{2 m_{N}^{2}}+\frac{E_{N}^{2}}{4 m_{N}}-\frac{m_{N}}{4}\right), \quad(\mathrm{A} 1) \\
\Pi_{V}^{k k}\left(\Gamma^{0}, \vec{q}\right)= & A_{20} C\left(\frac{E_{N}}{8}+\frac{m_{N}}{8}+\frac{q_{k}^{2}}{4 m_{N}}\right)=0, \\
& +B_{20} C\left(-\frac{E_{N}^{2}}{16 m_{N}}+\frac{m_{N}}{16}-\frac{q_{k}^{2} E_{N}}{8 m_{N}^{2}}+\frac{q_{k}^{2}}{8 m_{N}}\right) \\
& +C_{20} C\left(-\frac{E_{N}^{2}}{4 m_{N}}+\frac{m_{N}}{4}+\frac{q_{k}^{2} E_{N}}{2 m_{N}^{2}}+\frac{q_{k}^{2}}{2 m_{N}}\right), \quad(\mathrm{A} 3 \\
\Pi_{V}^{k k}\left(\Gamma^{n}, \vec{q}\right)= & A_{20} C\left(i \frac{\epsilon_{k n 0 \rho} q_{k} q_{\rho}}{4 m_{N}}\right)+B_{20} C\left(i \frac{\epsilon_{k n 0 \rho} q_{k} q_{\rho}}{4 m_{N}}\right),
\end{aligned}
$$

$$
\begin{gathered}
\Pi_{V}^{k 0}\left(\Gamma^{0}, \vec{q}\right)=A_{20} C\left(-i \frac{q_{k}}{4}-i \frac{q_{k} E_{N}}{4 m_{N}}\right) \\
+B_{20} C\left(-i \frac{q_{k}}{8}+i \frac{q_{k} E_{N}^{2}}{8 m_{N}^{2}}\right) \\
+C_{20} C\left(i \frac{q_{k}}{2}-i \frac{q_{k} E_{N}^{2}}{2 m_{N}^{2}}\right), \\
\Pi_{V}^{k 0}\left(\Gamma^{n}, \vec{q}\right)=A_{20} C\left(\epsilon_{k 0 n \rho}\left(\frac{q_{\rho}}{8}+\frac{q_{\rho} E_{N}}{8 m_{N}}\right)\right) \\
+B_{20} C\left(\epsilon_{k 0 n \rho}\left(\frac{q_{\rho}}{8}+\frac{q_{\rho} E_{N}}{8 m_{N}}\right)\right), \\
\Pi_{V}^{k j}\left(\Gamma^{0}, \vec{q}\right)=A_{20} C \frac{q_{k} q_{j}}{4 m_{N}}+B_{20} C\left(-\frac{q_{k} q_{j} E_{N}}{8 m_{N}^{2}}+\frac{q_{k} q_{j}}{8 m_{N}}\right) \\
+C_{20} C\left(\frac{q_{k} q_{j} E_{N}}{2 m_{N}^{2}}+\frac{q_{k} q_{j}}{2 m_{N}}\right), \\
\Pi_{V}^{k j}\left(\Gamma^{n}, \vec{q}\right)=A_{20} C\left(i \frac{\epsilon_{k n 0 \rho} q_{j} q_{\rho}}{8 m_{N}}+i \frac{\epsilon_{j n 0 \rho} q_{k} q_{\rho}}{8 m_{N}}\right) \\
+B_{20} C\left(i \frac{\epsilon_{k n 0 \rho} q_{j} q_{\rho}}{8 m_{N}}+i \frac{\epsilon_{j n 0 \rho} q_{k} q_{\rho}}{8 m_{N}}\right) .
\end{gathered}
$$

Axial-Vector:

$$
\Pi_{A}^{\mu \nu}\left(\Gamma^{0}, \vec{q}\right)=0,
$$

$$
\begin{aligned}
\Pi_{A}^{k 0}\left(\Gamma^{n}, \vec{q}\right)= & \tilde{A}_{20} C\left(-i \delta_{n k}\left(\frac{E_{N}}{4}+\frac{E_{N}^{2}}{8 m_{N}}+\frac{m_{N}}{8}\right)-i \frac{q_{k} q_{n}}{8 m_{N}}\right) \\
& +\tilde{B}_{20} C\left(i \frac{q_{k} q_{n} E_{N}}{8 m_{N}^{2}}\right), \\
\Pi_{A}^{k j}\left(\Gamma^{n}, \vec{q}\right)= & \tilde{A}_{20} C\left(\delta_{n j}\left(\frac{q_{k}}{8}+\frac{q_{k} E_{N}}{8 m_{N}}\right)+\delta_{n k}\left(\frac{q_{j}}{8}+\frac{q_{j} E_{N}}{8 m_{N}}\right)\right) \\
& +\tilde{B}_{20} C\left(-\frac{q_{k} q_{j} q_{n}}{8 m_{N}^{2}}\right),
\end{aligned}
$$

where $C=\sqrt{2 m_{N}^{2} /\left(E_{N}\left(E_{N}+m_{N}\right)\right)} ; E_{N}^{2}=m_{N}^{2}+\vec{q}^{2}$; the Latin indices $k, j$, and $n$ denote spatial directions 1,2 , and 3 ; and $k \neq j$. A summation is implied over the index $\rho$.

\section{APPENDIX B: CHIRAL PERTURBATION THEORY RESULTS}

For convenience, we collect in this Appendix the results of the $\mathrm{HB} \chi \mathrm{PT}$ results taken from Ref. [49] for the isovector $(I=1)$ and isoscalar $(I=0)$ first moments and axial charge: 


$$
\begin{aligned}
\tilde{A}_{20}^{I=1}(0)= & \tilde{A}_{20}^{I=1(0)}\left\{1-\frac{m_{\pi}^{2}}{\left(4 \pi f_{\pi}\right)^{2}}\left[\left(2 g_{A}^{2}+1\right) \ln \frac{m_{\pi}^{2}}{\lambda^{2}}+g_{A}^{2}\right]\right\} \\
& +\tilde{A}_{20}^{I=1(2, m)} m_{\pi}^{2}, \\
\tilde{B}_{20}^{I=1}(0)= & \tilde{B}_{20}^{I=1(0)}\left\{1-\frac{m_{\pi}^{2}}{\left(4 \pi f_{\pi}\right)^{2}}\left[\left(2 g_{A}^{2}+1\right) \ln \frac{m_{\pi}^{2}}{\lambda^{2}}+g_{A}^{2}\right]\right\} \\
& +\tilde{A}_{20}^{I=1(0)} \frac{m_{\pi}^{2} g_{A}^{2}}{3\left(4 \pi f_{\pi}\right)^{2}} \ln \frac{m_{\pi}^{2}}{\lambda^{2}}+\tilde{B}_{20}^{I=1(2, m)} m_{\pi}^{2}, \quad(\mathrm{~B} 1) \\
& +\tilde{A}_{10}^{I=1(2, m)} m_{\pi}^{2}, \\
\tilde{A}_{10}^{I=1}(0)= & \tilde{A}_{10}^{I=1(0)}\left\{1-\frac{m_{\pi}^{2}}{\left(4 \pi f_{\pi}\right)^{2}}\left[\left(2 g_{A}^{2}+1\right) \ln \frac{m_{\pi}^{2}}{\lambda^{2}}+g_{A}^{2}\right]\right\} \\
& +A_{20}^{I=1(2, m)} m_{\pi}^{2}, \\
A_{20}^{I=1}(0)= & A_{20}^{I=1(0)}\left\{1-\frac{m_{\pi}^{2}}{\left(4 \pi f_{\pi}\right)^{2}}\left[\left(3 g_{A}^{2}+1\right) \ln \frac{m_{\pi}^{2}}{\lambda^{2}}+2 g_{A}^{2}\right]\right\} \\
& +A_{20}^{I=1(0)} \frac{m_{\pi}^{2} g_{A}^{2}}{\left(4 \pi f_{\pi}\right)^{2}} \ln \frac{m_{\pi}^{2}}{\lambda^{2}}+B_{20}^{I=1(2, m)} m_{\pi}^{2}, \quad(\mathrm{~B} 3) \\
B_{20}^{I=1}(0)= & B_{20}^{I=1(0)}\left\{1-\frac{m_{\pi}^{2}}{\left(4 \pi f_{\pi}\right)^{2}}\left[\left(2 g_{A}^{2}+1\right) \ln \frac{m_{\pi}^{2}}{\lambda^{2}}+2 g_{A}^{2}\right]\right\} \\
& \\
&
\end{aligned}
$$$$
A_{20}^{I=0}(0)=A_{20}^{I=0(0)}+A_{20}^{I=0(2, m)} m_{\pi}^{2},
$$$$
B_{20}^{I=0}(0)=B_{20}^{I=0(0)}\left[1-\frac{3 g_{A}^{2} m_{\pi}^{2}}{\left(4 \pi f_{\pi}\right)^{2}} \ln \frac{m_{\pi}^{2}}{\lambda^{2}}\right]
$$$$
-A_{20}^{I=0(0)} \frac{3 g_{A}^{2} m_{\pi}^{2}}{\left(4 \pi f_{\pi}\right)^{2}} \ln \frac{m_{\pi}^{2}}{\lambda^{2}}+B_{20}^{I=0(2, m)} m_{\pi}^{2}
$$$$
+B_{20}^{I=0(2, \pi)}(0) \text {, }
$$$$
\tilde{A}_{10}^{I=0}(0)=\tilde{A}_{10}^{I=0(0)}\left\{1-\frac{3 g_{A}^{2} m_{\pi}^{2}}{\left(4 \pi f_{\pi}\right)^{2}}\left[\ln \frac{m_{\pi}^{2}}{\lambda^{2}}+1\right]\right\}+\tilde{A}_{10}^{I=0(2, m)} m_{\pi}^{2} .
$$

$$
\begin{aligned}
& A_{20}^{I=0}(0)=a_{20}^{s}+4 m_{\pi}^{2} \frac{c_{9}}{M_{0}^{2}}-\frac{3 a_{20}^{s} g_{A}^{2} m_{\pi}^{2}}{\left(4 \pi f_{\pi}\right)^{2}}\left[\frac{m_{\pi}^{2}}{M_{0}^{2}}+\frac{m_{\pi}^{2}}{M_{0}^{2}}\left(2-\frac{m_{\pi}^{2}}{M_{0}^{2}}\right) \ln \frac{m_{\pi}}{M_{0}}\right. \\
& \left.+\frac{m_{\pi}}{\sqrt{4 M_{0}^{2}-m_{\pi}^{2}}}\left(2-4 \frac{m_{\pi}^{2}}{M_{0}^{2}}+\frac{m_{\pi}^{4}}{M_{0}^{4}}\right) \arccos \left(\frac{m_{\pi}}{2 M_{0}}\right)\right]+\mathcal{O}\left(p^{3}\right) \\
& A_{20}^{I=1}(0)=a_{20}^{v}+\frac{a_{20}^{v} m_{\pi}^{2}}{\left(4 \pi f_{\pi}\right)^{2}}\left[-\left(3 g_{A}^{2}+1\right) \ln \frac{m_{\pi}^{2}}{\lambda^{2}}-2 g_{A}^{2}+g_{A}^{2} \frac{m_{\pi}^{2}}{M_{0}^{2}}\left(1+3 \ln \frac{m_{\pi}^{2}}{M_{0}^{2}}\right)-\frac{1}{2} g_{A}^{2} \frac{m_{\pi}^{4}}{M_{0}^{4}} \ln \frac{m_{\pi}^{2}}{M_{0}^{2}}\right. \\
& \left.+g_{A}^{2} \frac{m_{\pi}}{\sqrt{4 M_{0}^{2}-m_{\pi}^{2}}}\left(14-8 \frac{m_{\pi}^{2}}{M_{0}^{2}}+\frac{m_{\pi}^{4}}{M_{0}^{4}}\right) \arccos \left(\frac{m_{\pi}}{2 M_{0}}\right)\right]+\frac{\Delta a_{20}^{v}(0) g_{A} m_{\pi}^{2}}{3\left(4 \pi f_{\pi}\right)^{2}}\left[2 \frac{m_{\pi}^{2}}{M_{0}^{2}}\left(1+3 \ln \frac{m_{\pi}^{2}}{M_{0}^{2}}\right)\right. \\
& \left.-\frac{m_{\pi}^{4}}{M_{0}^{4}} \ln \frac{m_{\pi}^{2}}{M_{0}^{2}}+\frac{2 m_{\pi}\left(4 M_{0}^{2}-m_{\pi}^{2}\right)^{3 / 2}}{M_{0}^{4}} \arccos \left(\frac{m_{\pi}}{2 M_{0}}\right)\right]+4 m_{\pi}^{2} \frac{c_{8}^{(\lambda)}}{M_{0}^{2}}+\mathcal{O}\left(p^{3}\right), \\
& B_{20}^{I=1}(0)=b_{20}^{v} \frac{M_{N}\left(m_{\pi}\right)}{M_{0}}+\frac{a_{20}^{v} g_{A}^{2} m_{\pi}^{2}}{\left(4 \pi f_{\pi}\right)^{2}}\left[\left(3+\ln \frac{m_{\pi}^{2}}{M_{0}^{2}}\right)-\frac{m_{\pi}^{2}}{M_{0}^{2}}\left(2+3 \ln \frac{m_{\pi}^{2}}{M_{0}^{2}}\right)+\frac{m_{\pi}^{4}}{M_{0}^{4}} \ln \frac{m_{\pi}^{2}}{M_{0}^{2}}\right. \\
& \left.-\frac{2 m_{\pi}}{\sqrt{4 M_{0}^{2}-m_{\pi}^{2}}}\left(5-5 \frac{m_{\pi}^{2}}{M_{0}^{2}}+\frac{m_{\pi}^{4}}{M_{0}^{4}}\right) \arccos \left(\frac{m_{\pi}}{2 M_{0}}\right)\right]+\mathcal{O}\left(p^{3}\right), \\
& C_{20}^{I=1}(0)=c_{20}^{v} \frac{M_{N}\left(m_{\pi}\right)}{M_{0}}+\frac{a_{20}^{v} g_{A}^{2} m_{\pi}^{2}}{12\left(4 \pi f_{\pi}\right)^{2}}\left[-1+2 \frac{m_{\pi}^{2}}{M_{0}^{2}}\left(1+\ln \frac{m_{\pi}^{2}}{M_{0}^{2}}\right)-\frac{m_{\pi}^{4}}{M_{0}^{4}} \ln \frac{m_{\pi}^{2}}{M_{0}^{2}}\right. \\
& \left.+\frac{2 m_{\pi}}{\sqrt{4 M_{0}^{2}-m_{\pi}^{2}}}\left(2-4 \frac{m_{\pi}^{2}}{M_{0}^{2}}+\frac{m_{\pi}^{4}}{M_{0}^{4}}\right) \arccos \left(\frac{m_{\pi}}{2 M_{0}}\right)\right]+\mathcal{O}\left(p^{3}\right),
\end{aligned}
$$

We note that the expressions for $A_{20}^{I=1}$ and $\tilde{A}_{20}^{I=1}$ are the same as those given in Eq. (22) (up to a redefinition of $C, \tilde{C}$ and $\left.c_{8}, \tilde{c}_{8}\right)$. We have included them here using the notation of Ref. [49] for completeness. We performed a combined fit to the following $\mathrm{CB} \chi \mathrm{PT}$ results taken from Ref. [47]: 


$$
\begin{gathered}
B_{20}^{I=0}(0)=b_{20}^{s} \frac{M_{N}\left(m_{\pi}\right)}{M_{0}}-\frac{3 a_{20}^{s} g_{A}^{2} m_{\pi}^{2}}{\left(4 \pi f_{\pi}\right)^{2}}\left[\left(3+\ln \frac{m_{\pi}^{2}}{M_{0}^{2}}\right)-\frac{m_{\pi}^{2}}{M_{0}^{2}}\left(2+3 \ln \frac{m_{\pi}^{2}}{M_{0}^{2}}\right)+\frac{m_{\pi}^{4}}{M_{0}^{4}} \ln \frac{m_{\pi}^{2}}{M_{0}^{2}}\right. \\
\left.-\frac{2 m_{\pi}}{\sqrt{4 M_{0}^{2}-m_{\pi}^{2}}}\left(5-5 \frac{m_{\pi}^{2}}{M_{0}^{2}}+\frac{m_{\pi}^{4}}{M_{0}^{4}}\right) \arccos \left(\frac{m_{\pi}}{2 M_{0}}\right)\right]+\mathcal{O}\left(p^{3}\right), \\
C_{20}^{I=0}(0)=c_{20}^{s} \frac{M_{N}\left(m_{\pi}\right)}{M_{0}}-\frac{a_{20}^{s} g_{A}^{2} m_{\pi}^{2}}{4\left(4 \pi f_{\pi}\right)^{2}}\left[-1+2 \frac{m_{\pi}^{2}}{M_{0}^{2}}\left(1+\ln \frac{m_{\pi}^{2}}{M_{0}^{2}}\right)-\frac{m_{\pi}^{4}}{M_{0}^{4}} \ln \frac{m_{\pi}^{2}}{M_{0}^{2}}\right. \\
\left.+\frac{2 m_{\pi}}{\sqrt{4 M_{0}^{2}-m_{\pi}^{2}}}\left(2-4 \frac{m_{\pi}^{2}}{M_{0}^{2}}+\frac{m_{\pi}^{4}}{M_{0}^{4}}\right) \arccos \left(\frac{m_{\pi}}{2 M_{0}}\right)\right]+\mathcal{O}\left(p^{3}\right),
\end{gathered}
$$

\begin{tabular}{|c|c|c|c|c|c|}
\hline$m_{\pi}(\mathrm{GeV})$ (no. configurations) & $(Q)^{2}$ & $\begin{array}{c}A_{20} \\
\beta=3.9,24^{3} \times 48\end{array}$ & $B_{20}$ & $\tilde{A}_{20}$ & $\tilde{B}_{20}$ \\
\hline 0.4675 (477) & $\begin{array}{l}0.0 \\
0.322 \\
0.619(1) \\
0.897(2) \\
1.157(3) \\
1.404(4) \\
1.640(6)\end{array}$ & $\begin{array}{l}0.256(9) \\
0.230(7) \\
0.202(9) \\
0.178(12) \\
0.172(19) \\
0.154(22) \\
0.136(40)\end{array}$ & $\begin{array}{l}0.364(23) \\
0.337(18) \\
0.299(16) \\
0.277(22) \\
0.249(34) \\
0.232(34) \\
0.208(60)\end{array}$ & $\begin{array}{l}0.307(6) \\
0.273(5) \\
0.252(7) \\
0.227(11) \\
0.176(17) \\
0.191(22) \\
0.193(51)\end{array}$ & $\begin{array}{l}0.651(99) \\
0.487(121) \\
0.568(72) \\
0.456(69) \\
0.078(96) \\
0.319(65) \\
0.346(126)\end{array}$ \\
\hline $0.4319(365)$ & $\begin{array}{l}0.0 \\
0.321 \\
0.615(1) \\
0.888(3) \\
1.143(4) \\
1.385(6) \\
1.614(8)\end{array}$ & $\begin{array}{l}0.257(10) \\
0.219(8) \\
0.185(9) \\
0.167(13) \\
0.168(26) \\
0.141(29) \\
0.103(48)\end{array}$ & $\begin{array}{l}0.418(34) \\
0.361(29) \\
0.296(26) \\
0.257(34) \\
0.209(42) \\
0.151(42) \\
0.118(63)\end{array}$ & $\begin{array}{l}0.310(7) \\
0.264(7) \\
0.232(9) \\
0.211(14) \\
0.175(25) \\
0.173(37) \\
0.136(67)\end{array}$ & $\begin{array}{l}0.516(109) \\
0.431(155) \\
0.378(75) \\
0.339(86) \\
0.191(115) \\
0.242(99) \\
0.139(112)\end{array}$ \\
\hline $0.3770(553)$ & $\begin{array}{l}0.0 \\
0.320 \\
0.613(1) \\
0.884(3) \\
1.138(4) \\
1.377(6) \\
1.604(8)\end{array}$ & $\begin{array}{l}0.258(10) \\
0.217(10) \\
0.192(11) \\
0.164(20) \\
0.204(76) \\
0.166(59) \\
0.105(92)\end{array}$ & $\begin{array}{l}0.408(44) \\
0.360(32) \\
0.338(26) \\
0.265(40) \\
0.339(129) \\
0.271(97) \\
0.140(126)\end{array}$ & $\begin{array}{l}0.296(8) \\
0.266(7) \\
0.253(8) \\
0.237(26) \\
0.288(91) \\
0.218(70) \\
0.143(113)\end{array}$ & $\begin{array}{l}0.683(150) \\
0.878(170) \\
0.417(89) \\
0.349(132) \\
0.661(301) \\
0.402(176) \\
0.215(210)\end{array}$ \\
\hline 0.3032 (943) & $\begin{array}{l}0.0 \\
0.317(1) \\
0.601(2) \\
0.862(4) \\
1.103(6) \\
1.330(8) \\
1.543(10)\end{array}$ & $\begin{array}{l}0.255(16) \\
0.239(13) \\
0.199(13) \\
0.174(23) \\
0.135(27) \\
0.098(21) \\
0.105(34)\end{array}$ & $\begin{array}{l}0.309(40) \\
0.241(36) \\
0.268(32) \\
0.178(37) \\
0.156(45) \\
0.142(34) \\
0.097(40)\end{array}$ & $\begin{array}{l}0.284(10) \\
0.268(10) \\
0.223(11) \\
0.195(20) \\
0.172(24) \\
0.135(22) \\
0.087(27)\end{array}$ & $\begin{array}{l}0.277(136) \\
0.380(184) \\
0.048(104) \\
0.245(110) \\
0.304(118) \\
0.180(70) \\
0.051(75)\end{array}$ \\
\hline
\end{tabular}

where $M_{0}$ is the mass of the nucleon at the chiral limit.

\section{APPENDIX C: NUMERICAL RESULTS FOR THE ISOVECTOR SECTOR}

TABLE III. Results on $A_{20}$ and $B_{20}$ form factors at $\beta=3.9$; lattice size: $24^{3} \times 48$. 
TABLE IV. Results on $A_{20}$ and $B_{20}$ form factors at $\beta=3.9$; lattice size: $32^{3} \times 64$.

\begin{tabular}{|c|c|c|c|c|c|}
\hline$m_{\pi}(\mathrm{GeV})$ (no. configurations) & $(Q)^{2}$ & $\begin{aligned} & A_{20} \\
\beta= & 3.9,32^{3} \times 64\end{aligned}$ & $B_{20}$ & $\tilde{A}_{20}$ & $\tilde{B}_{20}$ \\
\hline \multirow[t]{9}{*}{$0.2978(351)$} & 0.0 & $0.243(15)$ & $0.375(67)$ & $0.287(10)$ & $0.413(95)$ \\
\hline & 0.183 & $0.230(14)$ & $0.382(67)$ & $0.262(8)$ & $0.454(215)$ \\
\hline & $0.354(1)$ & $0.207(14)$ & $0.318(54)$ & $0.244(7)$ & $0.282(98)$ \\
\hline & $0.516(1)$ & $0.196(14)$ & $0.298(50)$ & $0.228(9)$ & $0.298(100)$ \\
\hline & $0.670(2)$ & $0.164(20)$ & $0.249(61)$ & $0.228(15)$ & $0.441(138)$ \\
\hline & $0.817(3)$ & $0.165(16)$ & $0.206(44)$ & $0.204(11)$ & $0.318(65)$ \\
\hline & $0.957(4)$ & $0.154(18)$ & $0.188(40)$ & $0.179(14)$ & $0.165(64)$ \\
\hline & $1.222(6)$ & $0.145(28)$ & $0.173(55)$ & $0.191(38)$ & $0.252(110)$ \\
\hline & $1.348(7)$ & $0.095(23)$ & $0.170(42)$ & $0.151(36)$ & $0.123(81)$ \\
\hline \multirow[t]{9}{*}{$0.2600(667)$} & 0.0 & $0.263(13)$ & $0.301(47)$ & $0.275(13)$ & $0.752(174)$ \\
\hline & 0.182 & $0.240(09)$ & $0.284(52)$ & $0.261(10)$ & $0.666(331)$ \\
\hline & $0.352(1)$ & $0.222(10)$ & $0.252(38)$ & $0.259(11)$ & $0.582(164)$ \\
\hline & $0.512(1)$ & $0.196(12)$ & $0.221(40)$ & $0.256(16)$ & $0.524(158)$ \\
\hline & $0.664(2)$ & $0.185(17)$ & $0.246(48)$ & $0.216(19)$ & $0.263(185)$ \\
\hline & $0.808(3)$ & $0.161(14)$ & $0.233(34)$ & $0.217(16)$ & $0.378(105)$ \\
\hline & $0.945(4)$ & $0.143(15)$ & $0.204(34)$ & $0.181(18)$ & $0.247(93)$ \\
\hline & $1.205(6)$ & $0.100(22)$ & $0.122(42)$ & $0.127(24)$ & $0.140(128)$ \\
\hline & $1.328(7)$ & $0.106(22)$ & $0.162(40)$ & $0.124(26)$ & $0.072(105)$ \\
\hline
\end{tabular}

TABLE V. Results on $A_{20}$ and $B_{20}$ form factors at $\beta=4.05$.

\begin{tabular}{|c|c|c|c|c|c|}
\hline$m_{\pi}(\mathrm{GeV})$ (no. configurationss) & $(Q)^{2}$ & $\begin{array}{c}A_{20} \\
\beta=4.05,32^{3} \times 64\end{array}$ & $B_{20}$ & $\tilde{A}_{20}$ & $\tilde{B}_{20}$ \\
\hline \multirow[t]{9}{*}{0.4653 (419) } & 0.0 & $0.258(9)$ & $0.431(26)$ & $0.303(7)$ & $0.579(92)$ \\
\hline & 0.294 & $0.231(7)$ & $0.382(22)$ & $0.270(6)$ & $0.458(166)$ \\
\hline & $0.568(1)$ & $0.210(7)$ & $0.329(20)$ & $0.248(7)$ & $0.523(73)$ \\
\hline & $0.824(2)$ & $0.197(12)$ & $0.283(25)$ & $0.232(11)$ & $0.342(77)$ \\
\hline & $1.067(3)$ & $0.170(19)$ & $0.313(41)$ & $0.221(20)$ & $0.263(116)$ \\
\hline & $1.297(4)$ & $0.166(19)$ & $0.256(30)$ & $0.211(21)$ & $0.367(75)$ \\
\hline & $1.517(5)$ & $0.156(31)$ & $0.208(43)$ & $0.181(33)$ & $0.256(76)$ \\
\hline & $1.930(7)$ & $0.084(29)$ & $0.085(34)$ & $0.111(30)$ & $0.190(92)$ \\
\hline & $2.126(9)$ & $0.056(58)$ & $0.054(63)$ & $0.066(65)$ & $0.114(139)$ \\
\hline \multirow[t]{9}{*}{$0.4032(326)$} & 0.0 & $0.244(12)$ & $0.465(46)$ & $0.312(10)$ & $0.625(114)$ \\
\hline & 0.293 & $0.240(10)$ & $0.434(42)$ & $0.287(10)$ & $0.455(206)$ \\
\hline & $0.564(1)$ & $0.208(11)$ & $0.356(36)$ & $0.263(11)$ & $0.477(91)$ \\
\hline & $0.816(2)$ & $0.197(17)$ & $0.302(43)$ & $0.259(19)$ & $0.539(132)$ \\
\hline & $1.053(3)$ & $0.175(32)$ & $0.225(48)$ & $0.251(34)$ & $0.307(154)$ \\
\hline & $1.278(5)$ & $0.144(20)$ & $0.203(36)$ & $0.195(22)$ & $0.332(81)$ \\
\hline & $1.493(6)$ & $0.144(41)$ & $0.204(66)$ & $0.202(56)$ & $0.387(137)$ \\
\hline & $1.895(9)$ & $0.068(25)$ & $0.095(36)$ & $0.093(26)$ & $0.142(73)$ \\
\hline & $2.084(10)$ & $0.043(26)$ & $0.083(45)$ & $0.071(32)$ & $0.151(76)$ \\
\hline \multirow[t]{7}{*}{0.2925 (447) } & $0.0(0)$ & $0.231(24)$ & $0.426(67)$ & $0.310(23)$ & $0.283(268)$ \\
\hline & $0.291(1)$ & $0.237(20)$ & $0.307(66)$ & $0.279(15)$ & $0.328(322)$ \\
\hline & $0.556(2)$ & $0.216(21)$ & $0.343(47)$ & $0.252(18)$ & $0.459(202)$ \\
\hline & $0.801(3)$ & $0.236(41)$ & $0.279(69)$ & $0.223(33)$ & $0.025(207)$ \\
\hline & $1.029(5)$ & $0.136(39)$ & $0.116(77)$ & $0.165(34)$ & $0.028(238)$ \\
\hline & $1.245(7)$ & $0.139(72)$ & $0.090(84)$ & $0.219(95)$ & $0.484(266)$ \\
\hline & $1.450(10)$ & $0.104(42)$ & $0.132(67)$ & $0.157(52)$ & $0.273(129)$ \\
\hline
\end{tabular}


TABLE VI. Results on $A_{20}$ and $B_{20}$ form factors at $\beta=4.2$.

\begin{tabular}{|c|c|c|c|c|c|}
\hline$m_{\pi}(\mathrm{GeV})$ (no. configurations) & $(Q)^{2}$ & $\begin{array}{c}A_{20} \\
\beta=4.2,32^{3} \times 64\end{array}$ & $B_{20}$ & $\tilde{A}_{20}$ & $\tilde{B}_{20}$ \\
\hline \multirow[t]{9}{*}{$0.4698(357)$} & 0.0 & $0.252(13)$ & $0.402(35)$ & $0.295(9)$ & $0.768(126)$ \\
\hline & $0.467(1)$ & $0.215(12)$ & $0.343(29)$ & $0.272(8)$ & $0.606(145)$ \\
\hline & $0.886(2)$ & $0.205(14)$ & $0.264(29)$ & $0.260(11)$ & $0.595(95)$ \\
\hline & $1.270(4)$ & $0.214(27)$ & $0.220(47)$ & $0.249(27)$ & $0.523(141)$ \\
\hline & $1.627(6)$ & $0.140(37)$ & $0.240(78)$ & $0.178(47)$ & $0.030(159)$ \\
\hline & $1.961(8)$ & $0.131(36)$ & $0.127(40)$ & $0.177(39)$ & $0.226(113)$ \\
\hline & $2.276(10)$ & $0.118(29)$ & $0.057(25)$ & $0.114(28)$ & $0.146(83)$ \\
\hline & $2.861(14)$ & $0.056(40)$ & $0.017(43)$ & $0.054(28)$ & $0.058(100)$ \\
\hline & & $\beta=4.2,48^{3} \times 96$ & & & \\
\hline \multirow[t]{11}{*}{$0.2622(245)$} & 0.0 & $0.276(21)$ & $0.234(45)$ & $0.290(15)$ & $0.579(129)$ \\
\hline & 0.211 & $0.255(14)$ & $0.264(49)$ & $0.275(12)$ & $0.301(375)$ \\
\hline & $0.407(2)$ & $0.253(16)$ & $0.205(38)$ & $0.279(11)$ & $0.689(146)$ \\
\hline & $0.589(3)$ & $0.228(17)$ & $0.157(45)$ & $0.255(12)$ & $0.268(137)$ \\
\hline & $0.762(5)$ & $0.184(30)$ & $0.180(59)$ & $0.272(27)$ & $0.453(213)$ \\
\hline & $0.925(7)$ & $0.193(23)$ & $0.178(38)$ & $0.231(15)$ & $0.177(107)$ \\
\hline & $1.081(9)$ & $0.174(22)$ & $0.146(39)$ & $0.230(17)$ & $0.207(100)$ \\
\hline & $1.373(13)$ & $0.133(39)$ & $0.076(49)$ & $0.167(23)$ & $0.168(114)$ \\
\hline & $1.511(16)$ & $0.093(28)$ & $0.142(40)$ & $0.164(20)$ & $0.189(82)$ \\
\hline & $1.644(18)$ & $0.054(38)$ & $0.153(52)$ & $0.118(29)$ & $-0.001(95)$ \\
\hline & $1.773(20)$ & $0.026(37)$ & $0.138(62)$ & $0.152(40)$ & $0.031(107)$ \\
\hline
\end{tabular}

[1] P. Hagler et al. (LHPC), Phys. Rev. D 77, 094502 (2008).

[2] S. N. Syritsyn et al., Phys. Rev. D 81, 034507 (2010).

[3] D. Brommel et al. (QCDSF-UKQCD), Proc. Sci., LAT2007 (2007) 158.

[4] C. Alexandrou, Proc. Sci., LATTICE2010 (2010) 001.

[5] T. Yamazaki et al., Phys. Rev. D 79, 114505 (2009).

[6] C. Alexandrou et al. (ETM Collaboration), Phys. Rev. D 83, 045010 (2011).

[7] D. Müeller, D. Robaschik, B. Geyer, F. Dittes, and J. Hořejši, Fortschr. Phys. 42, 101 (1994).

[8] X.-D. Ji, Phys. Rev. D 55, 7114 (1997).

[9] A. Radyushkin, Phys. Rev. D 56, 5524 (1997).

[10] X.-D. Ji, Phys. Rev. Lett. 78, 610 (1997).

[11] S. Chekanov et al. (ZEUS Collaboration), J. High Energy Phys. 05 (2009) 108.

[12] F. Aaron et al. (H1 Collaboration), Phys. Lett. B 659, 796 (2008).

[13] A. Airapetian et al. (HERMES Collaboration), J. High Energy Phys. 11 (2009) 083.

[14] C. Muñoz Camacho et al. (Jefferson Lab Hall A Collaboration, Hall A DVCS Collaboration), Phys. Rev. Lett. 97, 262002 (2006).

[15] S. Stepanyan et al. (CLAS Collaboration), Phys. Rev. Lett. 87, 182002 (2001).

[16] M. Diehl, Phys. Rep. 388, 41 (2003).

[17] C. Alexandrou, M. Brinet, J. Carbonell, M. Constantinou, P. Harraud et al., Phys. Rev. D 83, 094502 (2011).

[18] R. Frezzotti, P. A. Grassi, S. Sint, and P. Weisz (Alpha Collaboration), J. High Energy Phys. 08 (2001) 058.

[19] R. Frezzotti and G. C. Rossi, J. High Energy Phys. 08 (2004) 007.

[20] P. Weisz, Nucl. Phys. B212, 1 (1983).
[21] A. Shindler, Phys. Rep. 461, 37 (2008).

[22] C. Alexandrou, arXiv:0906.4137.

[23] C. Alexandrou et al. (ETM Collaboration), Phys. Rev. D 80, 114503 (2009).

[24] V. Drach et al., Proc. Sci., LATTICE2008 (2008) 123.

[25] C. Alexandrou et al. (European Twisted Mass Collaboration), Phys. Rev. D 78, 014509 (2008).

[26] C. Alexandrou et al. (ETM Collaboration), Proc. Sci., LAT2007 (2007) 087.

[27] P. Dimopoulos, R. Frezzotti, C. Michael, G. C. Rossi, and C. Urbach, Phys. Rev. D 81, 034509 (2010).

[28] V. Drach et al., Proc. Sci., Lattice2010 (2010) 123.

[29] C. Alexandrou (ETM Collaboration), Proc. Sci., Lattice2010 (2010) 001.

[30] C. Alexandrou et al., Proc. Sci., LAT2009 (2009) 145.

[31] C. Alexandrou et al., Proc. Sci., LAT2008 (2008) 145.

[32] C. Alexandrou, S. Güsken, F. Jegerlehner, K. Schilling, and R. Sommer, Nucl. Phys. B414, 815 (1994).

[33] S. Güsken, Nucl. Phys. B, Proc. Suppl. 17, 361 (1990).

[34] D. Dolgov et al. (LHPC), Phys. Rev. D 66, 034506 (2002).

[35] W. Wilcox, T. Draper, and K.-F. Liu, Phys. Rev. D 46, 1109 (1992).

[36] M. Göckeler et al., Phys. Rev. D 54, 5705 (1996).

[37] C. Urbach, Proc. Sci., LAT2007 (2007) 022.

[38] C. Alexandrou, M. Constantinou, T. Korzec, H. Panagopoulos, and F. Stylianou, Phys. Rev. D 83, 014503 (2011).

[39] M. Göckeler et al., Nucl. Phys. B544, 699 (1999).

[40] M. Constantinou, V. Lubicz, H. Panagopoulos, and F. Stylianou, J. High Energy Phys. 10 (2009) 064.

[41] D. Pleiter et al. (QCDSF/UKQCD Collaboration), Proc. Sci., LATTICE2010 (2010) 153. 
[42] Y. Aoki et al., Phys. Rev. D 82, 014501 (2010).

[43] J. Bratt et al. (LHPC), Phys. Rev. D 82, 094502 (2010).

[44] D. B. Renner, Proc. Sci., LAT2009 (2009) 018.

[45] D. Arndt and M. J. Savage, Nucl. Phys. A697, 429 (2002).

[46] W. Detmold, W. Melnitchouk, and A. W. Thomas, Phys. Rev. D 66, 054501 (2002).

[47] M. Dorati, T. A. Gail, and T. R. Hemmert, Nucl. Phys. A798, 96 (2008).

[48] A. Airapetian et al. (HERMES Collaboration), Phys. Rev. D 75, 012007 (2007).

[49] M. Diehl, A. Manashov, and A. Schafer, Eur. Phys. J. A 31, 335 (2007).
[50] S. Alekhin, J. Blumlein, S. Klein, and S. Moch, Phys. Rev. D 81, 014032 (2010).

[51] A. Airapetian et al. (HERMES Collaboration), Phys. Lett. B 682, 345 (2010).

[52] J. Blumlein and H. Bottcher, Nucl. Phys. B841, 205 (2010).

[53] D. Brommel et al., Eur. Phys. J. Special Topics 162, 63 (2008).

[54] J. Pumplin, D. Stump, J. Huston, H. Lai, P. M. Nadolsky et al., J. High Energy Phys. 07 (2002) 012.

[55] A. D. Martin, R. G. Roberts, W. J. Stirling, and R. S. Thorne, Eur. Phys. J. C 28, 455 (2003). 